\title{
Generalized Fuzzy Bonferroni Harmonic Mean Operators and Their Applications in Group Decision Making
}

\author{
Jin Han Park and Eun Jin Park \\ Department of Applied Mathematics, Pukyong National University, Busan 608-737, Republic of Korea \\ Correspondence should be addressed to Jin Han Park; jihpark@pknu.ac.kr
}

Received 19 May 2013; Accepted 7 August 2013

Academic Editor: Deng-Feng Li

Copyright ( 2013 J. H. Park and E. J. Park. This is an open access article distributed under the Creative Commons Attribution License, which permits unrestricted use, distribution, and reproduction in any medium, provided the original work is properly cited.

\begin{abstract}
The Bonferroni mean (BM) operator is an important aggregation technique which reflects the correlations of aggregated arguments. Based on the BM and harmonic mean operators, H. Sun and M. Sun (2012) developed the fuzzy Bonferroni harmonic mean (FBHM) and fuzzy ordered Bonferroni harmonic mean (FOBHM) operators. In this paper, we study desirable properties of these operators and extend them, by considering the correlations of any three aggregated arguments instead of any two, to develop generalized fuzzy weighted Bonferroni harmonic mean (GFWBHM) operator and generalized fuzzy ordered weighted Bonferroni harmonic mean (GFOWBHM) operator. In particular, all these operators can be reduced to aggregate interval or real numbers. Then based on the GFWBHM and GFOWBHM operators, we present an approach to multiple attribute group decision making and illustrate it with a practical example.
\end{abstract}

\section{Introduction}

Multiple attribute group decision making (MAGDM) is the common phenomenon in modern life, which is to select the optimal alternative(s) from several alternatives or to get their ranking by aggregating the performances of each alternative under several attributes, in which the aggregation techniques play an important role. Considering the relationships among the aggregated arguments, we can classify the aggregation techniques into two categories: the ones which consider the aggregated arguments dependently and the others which consider the aggregated arguments independently. For the first category, the well-known ordered weighted averaging (OWA) operator $[1,2]$ is the representative, on the basis of which, a lot of generalizations have been developed, such as the ordered weighted geometric (OWG) operator [3-5], the ordered weighted harmonic mean (OWHM) operator [6], the continuous ordered weighted averaging (C-OWA) operator [7], the continuous ordered weighted geometric (C-OWG) operator [8]. The second category can reduce to two subcategories: the first subcategory focuses on changing the weight vector of the aggregation operators, such as the Choquet integral-based aggregation operators [9], in which the correlations of the aggregated arguments are measured subjectively by the decision makers, and the representatives of another subcategory are the power averaging (PA) operator [10] and the power geometric (PG) operator [11], both of which allow the aggregated arguments to support each other in aggregation process, on the basis of which the weighted vector is determined. The second subcategory focuses on the aggregated arguments such as the Bonferroni mean (BM) operator [12]. Yager [13] provided an interpretation of BM operator as involving a product of each argument with the average of the other arguments, a combined averaging and "anding" operator. Beliakov et al. [14] presented a composed aggregation technique called the generalized Bonferroni mean (GBM) operator, which models the average of the conjunctive expressions and the average of remaining. In fact, they extended the BM operator by considering the correlations of any three aggregated arguments instead of any two. However, both BM operator and the GBM operator ignore some aggregation information and the weight vector of the aggregated arguments. To overcome this drawback, Xia et al. [15] developed the generalized weighted Bonferroni mean (GWBM) operator as the weighted version of the GBM operator. Based on the GBM operator and geometric 
mean operator, they also developed the generalized Bonferoni geometric mean (GWBGM) operator. The fundamental characteristic of the GWBM operator is that it focuses on the group opinions, while the GWBGM operator gives more importance to the individual opinions. Because of the usefulness of the aggregation techniques, which reflect the correlations of arguments, most of them have been extended to fuzzy, intuitionistic fuzzy, or hesitant fuzzy environment [16-20].

Harmonic mean is the reciprocal of arithmetic mean of reciprocal, which is a conservative average to be used to provide for aggregation lying between the max and min operators, and is widely used as a tool to aggregate central tendency data [21]. In the existing literature, the harmonic mean is generally considered as a fusion technique of numerical data information. However, in many situations, the input arguments take the form of triangular fuzzy numbers because of time pressure, lack of knowledge, and people's limited expertise related with problem domain. Therefore, "how to aggregate fuzzy data by using the harmonic mean?" is an interesting research topic and is worth paying attention to. So $\mathrm{Xu}$ [21] developed the fuzzy harmonic mean operators such as fuzzy weighted harmonic mean (FWHM) operator, fuzzy ordered weighted harmonic mean (FOWHM) operator and fuzzy hybrid harmonic mean (FHHM) operator, and applied them to MAGDM. Wei [22] developed fuzzy induced ordered weighted harmonic mean (FIOWHM) operator and then, based on the FWHM and FIOWHM operators, presented the approach to MAGDM. H. Sun and M. Sun [23] further applied the BM operator to fuzzy environment, introduced the fuzzy Bonferroni harmonic mean (FBHM) operator and the fuzzy ordered Bonferroni harmonic mean (FOBHM) operator, and applied the FOBHM operator to multiple attribute decision making. In this paper, we will develop some new harmonic aggregation operators, including the generalized fuzzy weighted Bonferroni harmonic mean (GFWBHM) operator and generalized fuzzy ordered weighted Bonferroni harmonic mean (GFOWBHM) operator, and apply them to MAGDM.

In order to do this, the remainder of this paper is arranged in following sections. Section 2 first reviews some aggregation operators, including the BM, GBM, and GWBM operators. Then, some basic concepts related to triangular fuzzy numbers and some operational laws of triangular fuzzy numbers are introduced. The desirable properties of the FBHM and FOBHM operators are discussed. We extend them, by considering the correlations of any three aggregated arguments instead of any two, to develop generalized fuzzy weighted Bonferroni harmonic mean (GFWBHM) operator and generalized fuzzy ordered weighted Bonferroni harmonic mean (GFOWBHM) operator. In particular, all these operators can be reduced to aggregate interval or real numbers. Section 3 presents an approach to MAGDM based on the GFWBHM and GFOWBHM operators. Section 4 illustrates the presented approach with a practical example, verifies and shows the advantages of the presented approach, and makes a comparative study to the existing approaches. Section 5 ends the paper with some concluding remarks.

\section{Generalized Fuzzy Bonferroni Harmonic Mean Operators}

The Bonferroni mean operator was initially proposed by Bonferroni [12] and was also investigated intensively by Yager [13].

Definition 1. Let $p, q \geq 0$ and let $a_{i}(i=1,2, \ldots, n)$ be a collection of nonnegative numbers. If

$$
\operatorname{BM}^{p, q}\left(a_{1}, a_{2}, \ldots, a_{n}\right)=\left(\frac{1}{n(n-1)} \sum_{\substack{i, j=1 \\ i \neq j}}^{n} a_{i}^{p} a_{j}^{q}\right)^{1 /(p+q)},
$$

then $\mathrm{BM}^{p, q}$ is called the Bonferroni mean (BM) operator.

Beliakov et al. [14] further extended the BM operator by considering the correlations of any three aggregated arguments instead of any two.

Definition 2. Let $p, q, r \geq 0$ and let $a_{i}(i=1,2, \ldots, n)$ be a collection of nonnegative numbers. If

$$
\begin{aligned}
\operatorname{GBM}^{p, q, r} & \left(a_{1}, a_{2}, \ldots, a_{n}\right) \\
& =\left(\frac{1}{n(n-1)(n-2)} \sum_{\substack{i, j, k=1 \\
i \neq j \neq k}}^{n} a_{i}^{p} a_{j}^{q} a_{k}^{r}\right)^{1 /(p+q+r)},
\end{aligned}
$$

then $\mathrm{GBM}^{p, q, r}$ is called the generalized Bonferroni mean (GBM) operator.

In particular, if $r=0$, then the GBM operator reduces to the BM operator. However, it is noted that both BM operator and the GBM operator do not consider the situation that $i=j$ or $j=k$ or $i=k$, and the weight vector of the aggregated arguments is not also considered. To overcome this drawback, Xia et al. [15] defined the weighted version of the GBM operator.

Definition 3. Let $p, q, r \geq 0$ and let $a_{i}(i=1,2, \ldots, n)$ be a collection of nonnegative numbers with the weight vector $w=\left(w_{1}, w_{2}, \ldots, w_{n}\right)^{T}$ such that $w_{i}>0, i=1,2, \ldots, n$ and $\sum_{i=1}^{n} w_{i}=1$. If

$$
\begin{aligned}
\operatorname{GWBM}^{p, q, r}\left(a_{1}, a_{2}, \ldots, a_{n}\right) \\
=\left(\sum_{i, j, k=1}^{n} w_{i} w_{j} w_{k} a_{i}^{p} a_{j}^{q} a_{k}^{r}\right)^{1 /(p+q+r)},
\end{aligned}
$$

then $\mathrm{GWBM}^{p, q, r}$ is called the generalized weighted Bonferroni mean (GWBM) operator.

Some special cases can be obtained as the change of the parameters as follows. 
(1) If $r=0$, then the GWBM operator reduces to the following:

$$
\begin{aligned}
\operatorname{GWBM}^{p, q, 0}\left(a_{1}, a_{2}, \ldots, a_{n}\right) \\
=\left(\sum_{i, j, k=1}^{n} w_{i} w_{j} w_{k} a_{i}^{p} a_{j}^{q}\right)^{1 /(p+q)} \\
=\left(\sum_{i=1}^{n} w_{i} w_{j} a_{i}^{p} a_{j}^{q} \sum_{k=1}^{n} w_{k}\right)^{1 /(p+q)} \\
=\left(\sum_{i=1}^{n} w_{i} w_{j} a_{i}^{p} a_{j}^{q}\right)^{1 /(p+q)},
\end{aligned}
$$

which is the weighted Bonferroni mean (WBM) operator.

(2) If $q=0$ and $r=0$, then the GWBM operator reduces to the following:

$$
\begin{aligned}
\operatorname{GWBM}^{p, 0,0}\left(a_{1}, a_{2}, \ldots, a_{n}\right) \\
=\left(\sum_{i, j, k=1}^{n} w_{i} w_{j} w_{k} a_{i}^{p}\right)^{1 / p} \\
=\left(\sum_{i=1}^{n} w_{i} a_{i}^{p} \sum_{j=1}^{n} w_{j} \sum_{k=1}^{n} w_{k}\right)^{1 / p} \\
=\left(\sum_{i=1}^{n} w_{i} a_{i}^{p}\right)^{1 / p},
\end{aligned}
$$

which is the generalized weighted averaging operator. Furthermore, in this case, let us look at the GWBM operator for some special cases of $p$.

(1) If $p=1$, the GWBM operator reduces to the weighted averaging (WA) operator.

(2) If $p \rightarrow 0$, then the GWBM operator reduces to the weighted geometric (WG) operator.

(3) If $p \rightarrow+\infty$, then the GWBM operator reduces to the max operator.

The previous aggregation techniques can only deal with the situation that the arguments are represented by the exact numerical values, but are invalid if the aggregation information is given in other forms, such as triangular fuzzy number [24], which is a widely used tool to deal with uncertainty and fuzziness, described as follows.

Definition 4 (see [24]). A triangular fuzzy number $\widehat{a}$ can be defined by a triplet $\left[a^{L}, a^{M}, a^{U}\right]$. The membership function $\mu_{\hat{a}}(x)$ is defined as

$$
\mu_{\widehat{a}}(x)= \begin{cases}0, & x<a^{L} ; \\ \frac{x-a^{L}}{a^{M}-a^{L}}, & a^{L} \leq x \leq a^{M} ; \\ \frac{x-a^{U}}{a^{M}-a^{U}}, & a^{M} \leq x \leq a^{U} ; \\ 0, & x>a^{U},\end{cases}
$$

where $a^{U} \geq a^{M} \geq a^{L} \geq 0, a^{L}$, and $a^{U}$ stand for the lower and upper values of $\widehat{a}$, respectively, and $a^{M}$ stands for the modal value [24]. In particular, if any two of $a^{L}, a^{M}$, and $a^{U}$ are equal, then $\widehat{a}$ reduces to an interval number; if all $a^{L}, a^{M}$, and $a^{U}$ are equal, then $\widehat{a}$ reduces to a real number. For convenience, we let $\Omega$ be the set of all triangular fuzzy numbers.

Let $\widehat{a}=\left[a^{L}, a^{M}, a^{U}\right]$ and $\widehat{b}=\left[b^{L}, b^{M}, b^{U}\right]$ be two triangular fuzzy numbers, then some operational laws defined as follows [24]:

(1) $\widehat{a}+\widehat{b}=\left[a^{L}, a^{M}, a^{U}\right]+\left[b^{L}, b^{M}, b^{U}\right]=\left[a^{L}+b^{L}, a^{M}+\right.$ $\left.b^{M}, a^{U}+b^{U}\right]$

(2) $\lambda \widehat{a}=\lambda\left[a^{L}, a^{M}, a^{U}\right]=\left[\lambda a^{L}, \lambda a^{M}, \lambda a^{U}\right]$;

(3) $\widehat{a} \times \widehat{b}=\left[a^{L}, a^{M}, a^{U}\right] \times\left[b^{L}, b^{M}, b^{U}\right]=\left[a^{L} b^{L}, a^{M} b^{M}\right.$, $\left.a^{U} b^{U}\right]$

(4) $1 / \widehat{a}=1 /\left[a^{L}, a^{M}, a^{U}\right]=\left[1 / a^{U}, 1 / a^{M}, 1 / a^{L}\right]$.

In order to compare two triangular fuzzy numbers, $\mathrm{Xu}$ [21] provided the following definition.

Definition 5. Let $\widehat{a}=\left[a^{L}, a^{M}, a^{U}\right]$ and let $\widehat{b}=\left[b^{L}, b^{M}, b^{U}\right]$ be two triangular fuzzy numbers; then the degree of possibility of $\widehat{a} \geq \widehat{b}$ is defined as follows:

$$
\begin{aligned}
p(\widehat{a} \geq \widehat{b}) & \\
= & \delta \max \left\{1-\max \left(\frac{b^{M}-a^{L}}{a^{M}-a^{L}+b^{M}-b^{L}}, 0\right), 0\right\} \\
& +(1-\delta) \max \left\{1-\max \left(\frac{b^{U}-a^{M}}{a^{U}-a^{M}+b^{U}-b^{M}}, 0\right), 0\right\}, \\
& \delta \in[0,1],
\end{aligned}
$$

which satisfies the following properties:

$$
\begin{gathered}
0 \leq p(\widehat{a} \geq \widehat{b}) \leq 1, \quad p(\widehat{a} \geq \widehat{a})=0.5, \\
p(\widehat{a} \geq \widehat{b})+p(\widehat{b} \geq \widehat{a})=1 .
\end{gathered}
$$

Here, $\delta$ reflects the decision maker's risk-bearing attitude. If $\delta>0.5$, then the decision maker is risk lover; if $\delta=0.5$, then the decision maker is neutral to risk; if $\delta<0.5$, then the decision maker is risk avertor.

In the following, we will give a simple procedure for ranking of the triangular fuzzy numbers $\widehat{a}_{i}(i=1,2, \ldots, n)$. First, by using (7), we compare each $\widehat{a}_{i}$ with all the $\widehat{a}_{j}(j=$ $1,2, \ldots, n)$; for simplicity, let $p_{i j}=p\left(\widehat{a}_{i} \geq \widehat{a}_{j}\right)$, and then we develop a possibility matrix $[25,26]$ as

$$
P=\left(\begin{array}{cccc}
p_{11} & p_{12} & \cdots & p_{1 n} \\
p_{21} & p_{22} & \cdots & p_{2 n} \\
& & \vdots & \\
p_{n 1} & p_{n 2} & \cdots & p_{n n}
\end{array}\right),
$$

where $p_{i j} \geq 0, p_{i j}+p_{j i}=1, p_{i i}=1 / 2, i, j=1,2, \ldots, n$. 
Summing all elements in each line of matrix $P$, we have $p_{i}=\sum_{j=1}^{n} p_{i j}, i=1,2, \ldots, n$. Then, in accordance with the values of $p_{i}(i=1,2, \ldots, n)$, we rank the $\widehat{a}_{i}(i=1,2, \ldots, n)$ in descending order.

To aggregate the triangular fuzzy correlated information, based on the BM and weighted harmonic mean operators, H. Sun and M. Sun [23] developed the fuzzy Bonferroni harmonic mean operator. Because this operator considers the weight vector of the aggregated arguments, we redefine this operator as fuzzy weighted Bonferroni harmonic mean operator.

Definition 6 (see [23]). Let $\widehat{a}_{i}=\left[a_{i}^{L}, a_{i}^{M}, a_{i}^{U}\right](i=1,2, \ldots, n)$ be a collection of triangular fuzzy numbers, let $w=\left(w_{1}\right.$, $\left.w_{2}, \ldots, w_{n}\right)^{T}$ be the weight vector of $\widehat{a}_{i}(i=1,2, \ldots, n)$, where $w_{i}$ indicates the importance degree of $\widehat{a}_{i}$, satisfying $w_{i}>0$, $i=1,2, \ldots, n$ and $\sum_{i=1}^{n} w_{i}=1$. If

$$
\begin{aligned}
\operatorname{FWBHM}^{p, q}\left(\widehat{a}_{1}, \widehat{a}_{2}, \ldots, \widehat{a}_{n}\right) & \\
= & \frac{1}{\left(\sum_{i, j=1}^{n}\left(w_{i} w_{j} / \widehat{a}_{i}^{p} \widehat{a}_{j}^{q}\right)\right)^{1 /(p+q)}} \\
= & {\left[\frac{1}{\left(\sum_{i, j=1}^{n}\left(w_{i} w_{j} /\left(a_{i}^{L}\right)^{p}\left(a_{j}^{L}\right)^{q}\right)\right)^{1 /(p+q)}},\right.} \\
& \frac{1}{\left(\sum_{i, j=1}^{n}\left(w_{i} w_{j} /\left(a_{i}^{M}\right)^{p}\left(a_{j}^{M}\right)^{q}\right)\right)^{1 /(p+q)}}, \\
& \left.\frac{1}{\left(\sum_{i, j=1}^{n}\left(w_{i} w_{j} /\left(a_{i}^{U}\right)^{p}\left(a_{j}^{U}\right)^{q}\right)\right)^{1 /(p+q)}}\right],
\end{aligned}
$$

where $p, q \geq 0$, then $\mathrm{FWBHM}^{p, q}$ is called the fuzzy weighted Bonferroni harmonic mean (FWBHM) operator.

In particular, if $w=(1 / n, 1 / n, \ldots, 1 / n)^{T}$, then the FWBHM operator reduces to the following:

$$
\operatorname{FBHM}^{p, q}\left(\widehat{a}_{1}, \widehat{a}_{2}, \ldots, \widehat{a}_{n}\right)=\frac{1}{\left(\left(1 / n^{2}\right) \sum_{i, j=1}^{n}\left(1 / \widehat{a}_{i}^{p} \widehat{a}_{j}^{q}\right)\right)^{1 /(p+q)}},
$$

which we call the fuzzy Bonferroni harmonic mean (FBHM) operator.

In addition, a special case can obtained as the change of parameter. If $q=0$, then the FWBHM operator reduces to the following:

$$
\begin{aligned}
& \operatorname{FWBHM}^{p, 0}\left(\widehat{a}_{1}, \widehat{a}_{2}, \ldots, \widehat{a}_{n}\right) \\
& =\frac{1}{\left(\sum_{i, j=1}^{n}\left(w_{i} w_{j} / \widehat{a}_{i}^{p}\right)\right)^{1 / p}}=\frac{1}{\left(\sum_{i=1}^{n}\left(w_{i} / \widehat{a}_{i}^{p}\right) \sum_{j=1}^{n} w_{j}\right)^{1 / p}} \\
& =\frac{1}{\left(\sum_{i=1}^{n}\left(w_{i} / \widehat{a}_{i}^{p}\right)\right)^{1 / p}}
\end{aligned}
$$

$$
\begin{gathered}
=\left[\frac{1}{\left(\sum_{i=1}^{n}\left(w_{i} /\left(a_{i}^{L}\right)^{p}\right)\right)^{1 / p}}, \frac{1}{\left(\sum_{i=1}^{n}\left(w_{i} /\left(a_{i}^{M}\right)^{p}\right)\right)^{1 / p}},\right. \\
\left.\frac{1}{\left(\sum_{i=1}^{n}\left(w_{i} /\left(a_{i}^{U}\right)^{p}\right)\right)^{1 / p}}\right],
\end{gathered}
$$

which we call the fuzzy weighted generalized harmonic mean (FWGHM) operator.

On the basis of the operational laws of triangular fuzzy numbers, the FWBHM operator has the following properties.

Theorem 7. Let $p, q \geq 0$, and let $\widehat{a}_{i}=\left[a_{i}^{L}, a_{i}^{M}, a_{i}^{U}\right](i=$ $1,2, \ldots, n)$ be a collection of triangular fuzzy numbers, and the following are valid.

(1) Idempotency. If all $\widehat{a}_{i}(i=1,2, \ldots, n)$ are equal, that is, $\widehat{a}_{i}=$ $\widehat{a}$, for all $i$, then

$$
\operatorname{FWBHM}^{p, q}\left(\widehat{a}_{1}, \widehat{a}_{2}, \ldots, \widehat{a}_{n}\right)=\widehat{a} .
$$

(2) Boundedness. $\widehat{a}^{-} \leq \operatorname{FWBHM}^{p, q}\left(\widehat{a}_{1}, \widehat{a}_{2}, \ldots, \widehat{a}_{n}\right) \leq \widehat{a}^{+}$, where $\widehat{a}^{-}=\left[\min _{i}\left\{a_{i}^{L}\right\}, \min _{i}\left\{a_{i}^{M}\right\}, \min _{i}\left\{a_{i}^{U}\right\}\right]$ and $\widehat{a}^{+}=$ $\left[\max _{i}\left\{a_{i}^{L}\right\}, \max _{i}\left\{a_{i}^{M}\right\}, \max _{i}\left\{a_{i}^{U}\right\}\right]$.

(3) Commutativity. Let $\hat{a}_{i}^{\prime}=\left[a_{i}^{\prime L}, a_{i}^{\prime M}, a_{i}^{\prime U}\right](i=1,2, \ldots, n)$ be a collection of triangular fuzzy numbers, and then

$$
\begin{aligned}
& \operatorname{FWBHM}^{p, q}\left(\widehat{a}_{1}, \widehat{a}_{2}, \ldots, \widehat{a}_{n}\right) \\
& =\operatorname{FWBHM}^{p, q}\left(\widehat{a}_{1}^{\prime}, \widehat{a}_{2}^{\prime}, \ldots, \widehat{a}_{n}^{\prime}\right),
\end{aligned}
$$

where $\left(\hat{a}_{1}^{\prime}, \widehat{a}_{2}^{\prime}, \ldots, \hat{a}_{n}^{\prime}\right)$ is any permutation of $\left(\widehat{a}_{1}, \widehat{a}_{2}, \ldots, \widehat{a}_{n}\right)$.

Proof. Since (2) can be proven easily, we prove (1) and (3) as follows.

(1) Since $\widehat{a}_{i}=\widehat{a}$, we have

$$
\begin{aligned}
\operatorname{FWBHM}^{p, q}\left(\widehat{a}_{1}, \widehat{a}_{2}, \ldots, \widehat{a}_{n}\right) \\
=\frac{1}{\left(\sum_{i, j=1}^{n}\left(w_{i} w_{j} / \widehat{a}^{p} \widehat{a}^{q}\right)\right)^{1 /(p+q)}} \\
=\frac{1}{\left(\sum_{i, j=1}^{n}\left(w_{i} w_{j} / \widehat{a}^{p+q}\right)\right)^{1 /(p+q)}} \\
=\frac{\widehat{a}}{\left(\sum_{i=1}^{n} w_{i} \sum_{j=1}^{n} w_{j}\right)^{1 /(p+q)}} \\
=\widehat{a} .
\end{aligned}
$$


(3) Since $\left(\widehat{a}_{1}^{\prime}, \hat{a}_{2}^{\prime}, \ldots, \hat{a}_{n}^{\prime}\right)$ is any permutation of $\left(\widehat{a}_{1}, \widehat{a}_{2}\right.$, $\left.\ldots, \widehat{a}_{n}\right)$, then

$$
\begin{aligned}
\operatorname{FWBHM}^{p, q}\left(\widehat{a}_{1}, \widehat{a}_{2}, \ldots, \widehat{a}_{n}\right) \\
=\frac{1}{\left(\sum_{i, j=1}^{n}\left(w_{i} w_{j} / \widehat{a}_{i}^{p} \hat{a}_{j}^{q}\right)\right)^{1 /(p+q)}} \\
=\frac{1}{\left(\sum_{i, j=1}^{n}\left(w_{i} w_{j} /\left(\hat{a}_{i}^{\prime}\right)^{p}\left(\hat{a}_{j}^{\prime}\right)^{q}\right)\right)^{1 /(p+q)}} \\
=\operatorname{FWBHM}^{p, q}\left(\hat{a}_{1}^{\prime}, \hat{a}_{2}^{\prime}, \ldots, \hat{a}_{n}^{\prime}\right) .
\end{aligned}
$$

In particular, if the triangular fuzzy numbers $\widehat{a}_{i}=\left[a_{i}^{L}\right.$, $\left.a_{i}^{M}, a_{i}^{U}\right](i=1,2, \ldots, n)$ reduce to the interval numbers $\widetilde{a}_{i}=\left[a_{i}^{L}, a_{i}^{U}\right](i=1,2, \ldots, n)$, then the FWBHM operator (10) reduces to the uncertain weighted Bonferroni harmonic mean (UWBHM) operator as follows:

$$
\begin{aligned}
\operatorname{UWBHM}^{p, q}\left(\widetilde{a}_{1}, \widetilde{a}_{2}, \ldots, \widetilde{a}_{n}\right) \\
=\frac{1}{\left(\sum_{i, j=1}^{n}\left(w_{i} w_{j} / \widetilde{a}_{i}^{p} \widetilde{a}_{j}^{q}\right)\right)^{1 /(p+q)}} \\
=\left[\frac{1}{\left(\sum_{i, j=1}^{n}\left(w_{i} w_{j} /\left(a_{i}^{L}\right)^{p}\left(a_{j}^{L}\right)^{q}\right)\right)^{1 /(p+q)}},\right. \\
\left.\frac{1}{\left(\sum_{i, j=1}^{n}\left(w_{i} w_{j} /\left(a_{i}^{U}\right)^{p}\left(a_{j}^{U}\right)^{q}\right)\right)^{1 /(p+q)}}\right] .
\end{aligned}
$$

If $w=(1 / n, 1 / n, \ldots, 1 / n)^{T}$, then the UWBHM operator reduces to the uncertain Bonferroni harmonic mean (UBHM) operator as follows:

$$
\begin{aligned}
\operatorname{UBHM}^{p, q}\left(\widetilde{a}_{1}, \tilde{a}_{2}, \ldots, \tilde{a}_{n}\right) \\
=\frac{1}{\left(\left(1 / n^{2}\right) \sum_{i, j=1}^{n}\left(1 / \tilde{a}_{i}^{p} \widetilde{a}_{j}^{q}\right)\right)^{1 /(p+q)}} \\
=\left[\frac{1}{\left(\left(1 / n^{2}\right) \sum_{i, j=1}^{n}\left(1 /\left(a_{i}^{L}\right)^{p}\left(a_{j}^{L}\right)^{q}\right)\right)^{1 /(p+q)}},\right. \\
\\
\left.\quad \frac{1}{\left(\left(1 / n^{2}\right) \sum_{i, j=1}^{n}\left(1 /\left(a_{i}^{U}\right)^{p}\left(a_{j}^{U}\right)^{q}\right)\right)^{1 /(p+q)}}\right] .
\end{aligned}
$$

If $a_{i}^{L}=a_{i}^{U}=a_{i}$, for all $i$, then the UWBHM operator (17) reduces to the weighted Bonferroni harmonic mean (WBHM) operator as follows:

$$
\operatorname{WBHM}^{p, q}\left(a_{1}, a_{2}, \ldots, a_{n}\right)=\frac{1}{\left(\sum_{i, j=1}^{n}\left(w_{i} w_{j} / a_{i}^{p} a_{j}^{q}\right)\right)^{1 /(p+q)}} .
$$

In this case, if $w=(1 / n, 1 / n, \ldots, 1 / n)^{T}$, then the WBHM operator reduces to the Bonferroni harmonic mean (BHM) operator:

$$
\operatorname{BHM}^{p, q}\left(a_{1}, a_{2}, \ldots, a_{n}\right)=\frac{1}{\left(\left(1 / n^{2}\right) \sum_{i, j=1}^{n}\left(1 / a_{i}^{p} a_{j}^{q}\right)\right)^{1 /(p+q)}} .
$$

Example 8. Given a collection of triangular fuzzy numbers: $\widehat{a}_{1}=[2,3,4], \widehat{a}_{2}=[1,2,4], \widehat{a}_{3}=[2,4,6], \widehat{a}_{4}=[1,3,5]$, let $w=$ $(0.3,0.1,0.2,0.4)^{T}$ be the weight vector of $\widehat{a}_{i}(i=1,2,3,4)$; then, by FWBHM operator (10) (let $p=q=2$ ), we have

$$
\begin{gathered}
\operatorname{FWBHM}^{2,2}\left(\widehat{a}_{1}, \widehat{a}_{2}, \widehat{a}_{3}, \widehat{a}_{4}\right) \\
=\left[\frac{1}{\left(\sum_{i, j=1}^{4}\left(w_{i} w_{j} /\left(a_{i}^{L}\right)^{2}\left(a_{j}^{L}\right)^{2}\right)\right)^{1 / 4}},\right. \\
\frac{1}{\left(\sum_{i, j=1}^{4}\left(w_{i} w_{j} /\left(a_{i}^{M}\right)^{2}\left(a_{j}^{M}\right)^{2}\right)\right)^{1 / 4}}, \\
=[1.27,2.95,4.64] .
\end{gathered}
$$

Based on the OWA and FWBHM operators and Definition 5, we define fuzzy ordered weighted Bonferroni harmonic mean (FOWBHM) operator as follows.

Definition 9. Let $\widehat{a}_{i}=\left[a_{i}^{L}, a_{i}^{M}, a_{i}^{U}\right](i=1,2, \ldots, n)$ be a collection of triangular fuzzy numbers. For $p, q \geq 0$, a fuzzy ordered weighted Bonferroni harmonic mean (FOWBHM) operator of dimension $n$ is a mapping FOWBHM ${ }^{p, q}: \Omega^{n} \rightarrow$ $\Omega$, that has an associated vector $\omega=\left(\omega_{1}, \omega_{2}, \ldots, \omega_{n}\right)^{T}$ such that $\omega_{i} \geq 0$ and $\sum_{i=1}^{n} \omega_{i}=1$. Furthermore,

$$
\begin{aligned}
\operatorname{FOWBHM}^{p, q}\left(\widehat{a}_{1}, \hat{a}_{2}, \ldots, \widehat{a}_{n}\right) \\
=\frac{1}{\left(\sum_{i, j=1}^{n}\left(w_{i} w_{j} / \widehat{a}_{\sigma(i)}^{p} \hat{a}_{\sigma(j)}^{q}\right)\right)^{1 /(p+q)}} \\
=\left[\frac{1}{\left(\sum_{i, j=1}^{n}\left(w_{i} w_{j} /\left(a_{\sigma(i)}^{L}\right)^{p}\left(a_{\sigma(j)}^{L}\right)^{q}\right)\right)^{1 /(p+q)}},\right.
\end{aligned}
$$

$$
\begin{gathered}
\frac{1}{\left(\sum_{i, j=1}^{n}\left(w_{i} w_{j} /\left(a_{\sigma(i)}^{M}\right)^{p}\left(a_{\sigma(j)}^{M}\right)^{q}\right)\right)^{1 /(p+q)}}, \\
\left.\frac{1}{\left(\sum_{i, j=1}^{n}\left(w_{i} w_{j} /\left(a_{\sigma(i)}^{U}\right)^{p}\left(a_{\sigma(j)}^{U}\right)^{q}\right)\right)^{1 /(p+q)}}\right],
\end{gathered}
$$


where $\widehat{a}_{\sigma(i)}=\left[a_{\sigma(i)}^{L}, a_{\sigma(i)}^{M}, a_{\sigma(i)}^{U}\right](i=1,2, \ldots, n)$, and $(\sigma(1)$, $\sigma(2), \ldots, \sigma(n))$ is a permutation of $(1,2, \ldots, n)$ such that $\widehat{a}_{\sigma(i-1)} \geq \widehat{a}_{\sigma(i)}$ for all $i$.

However, if there is a tie between $\widehat{a}_{i}$ and $\widehat{a}_{j}$, then we replace each of $\widehat{a}_{i}$ and $\widehat{a}_{j}$ by their average $\left(\widehat{a}_{i}+\widehat{a}_{j}\right) / 2$ in process of aggregation. If $k$ items are tied, then we replace these by $k$ replicas of their average. The weighting vector $\omega=\left(\omega_{1}, \omega_{2}, \ldots, \omega_{n}\right)^{T}$ can be determined by using some weights determining methods like the normal distribution based method; see [27-29] for more details.

If $\omega=(1 / n, 1 / n, \ldots, 1 / n)^{T}$, then the FOWBHM operator reduces to the FBHM operator; in addition, if $q=0$, then the FOWBHM operator reduces to the following:

$$
\begin{aligned}
& \operatorname{FOWBHM}^{p, 0}\left(\widehat{a}_{1}, \widehat{a}_{2}, \ldots, \widehat{a}_{n}\right) \\
& =\frac{1}{\left(\sum_{i, j=1}^{n}\left(w_{i} w_{j} / \widehat{a}_{\sigma(i)}^{p}\right)\right)^{1 / p}}=\frac{1}{\left(\sum_{i=1}^{n}\left(w_{i} / \widehat{a}_{\sigma(i)}^{p}\right) \sum_{j=1}^{n} w_{j}\right)^{1 / p}} \\
& =\frac{1}{\left(\sum_{i=1}^{n}\left(w_{i} / \widehat{a}_{\sigma(i)}^{p}\right)\right)^{1 / p}} \\
& =\left[\frac{1}{\left(\sum_{i=1}^{n}\left(w_{i} /\left(a_{\sigma(i)}^{L}\right)^{p}\right)\right)^{1 / p}}, \frac{1}{\left(\sum_{i=1}^{n}\left(w_{i} /\left(a_{\sigma(i)}^{M}\right)^{p}\right)\right)^{1 / p}},\right. \\
& \left.\frac{1}{\left(\sum_{i=1}^{n}\left(w_{i} /\left(a_{\sigma(i)}^{U}\right)^{p}\right)\right)^{1 / p}}\right],
\end{aligned}
$$

which we call the fuzzy ordered weighted generalized harmonic mean (FOWGHM) operator.

In particular, if the triangular fuzzy numbers $\widehat{a}_{i}=$ $\left[a_{i}^{L}, a_{i}^{M}, a_{i}^{U}\right](i=1,2, \ldots, n)$ reduce to the interval numbers $\tilde{a}_{i}=\left[a_{i}^{L}, a_{i}^{U}\right](i=1,2, \ldots, n)$, then the FOWBHM operator reduces to the uncertain ordered weighted Bonferroni harmonic mean (UOWBHM) operator as follows:

$$
\begin{aligned}
& \operatorname{UOWBHM}^{p, q}\left(\widetilde{a}_{i}, \widetilde{a}_{2}, \ldots, \widetilde{a}_{n}\right) \\
& =\frac{1}{\left(\sum_{i, j=1}^{n}\left(\omega_{i} \omega_{j} / \tilde{a}_{\sigma(i)}^{p} \widetilde{a}_{\sigma(j)}^{q}\right)\right)^{1 /(p+q)}} \\
& =\left[\frac{1}{\left(\sum_{i, j=1}^{n}\left(w_{i} w_{j} /\left(a_{\sigma(i)}^{L}\right)^{p}\left(a_{\sigma(j)}^{L}\right)^{q}\right)\right)^{1 /(p+q)}},\right. \\
& \left.\frac{1}{\left(\sum_{i, j=1}^{n}\left(w_{i} w_{j} /\left(a_{\sigma(i)}^{U}\right)^{p}\left(a_{\sigma(j)}^{U}\right)^{q}\right)\right)^{1 /(p+q)}}\right],
\end{aligned}
$$

where $\tilde{a}_{\sigma(i)}=\left[a_{\sigma(i)}^{L}, a_{\sigma(i)}^{U}\right]$, and $(\sigma(1), \sigma(2), \ldots, \sigma(n))$ is a permutation of $(1,2, \ldots, n)$ such that $\tilde{a}_{\sigma(i-1)} \geq \tilde{a}_{\sigma(i)}$ for all $i$.
If there is a tie between $\tilde{a}_{i}$ and $\tilde{a}_{j}$, then we replace each of $\tilde{a}_{i}$ and $\widetilde{a}_{j}$ by their average $\left(\widetilde{a}_{i}+\widetilde{a}_{j}\right) / 2$ in process of aggregation. If $k$ items are tied, then we replace these by $k$ replicas of their average.

If $a_{i}^{L}=a_{i}^{U}=a_{i}$, for all $i$, then the UOWBHM operator reduces to the ordered weighted Bonferroni harmonic mean (OWBHM) operator as follows:

$$
\operatorname{OWBHM}^{p, q}\left(a_{1}, a_{2}, \ldots, a_{n}\right)=\frac{1}{\left(\sum_{i, j=1}^{n}\left(\omega_{i} \omega_{j} / b_{i}^{p} b_{j}^{q}\right)\right)^{1 /(p+q)}},
$$

where $b_{i}$ is the $i$ th largest of $a_{i}(i=1,2, \ldots, n)$. The OWBHM operator (25) has some special cases.

(1) If $\omega=(1,0, \ldots, 0)^{T}$, then

$$
\operatorname{OWBHM}^{p, q}\left(a_{1}, a_{2}, \ldots, a_{n}\right)=\max \left\{a_{i}\right\}=b_{1} \text {. }
$$

(2) If $\omega=(0,0, \ldots, 1)^{T}$, then

$$
\operatorname{OWBHM}^{p, q}\left(a_{1}, a_{2}, \ldots, a_{n}\right)=\min \left\{a_{i}\right\}=b_{n} .
$$

(3) If $\omega=(1 / n, 1 / n, \ldots, 1 / n)^{T}$, then

$$
\begin{aligned}
\operatorname{OWBHM}^{p, q}\left(a_{1}, a_{2}, \ldots, a_{n}\right) \\
=\frac{1}{\left(\left(1 / n^{2}\right) \sum_{i, j=1}^{n}\left(1 / b_{i}^{p} b_{j}^{q}\right)\right)^{1 /(p+q)}} \\
=\frac{1}{\left(\left(1 / n^{2}\right) \sum_{i, j=1}^{n}\left(1 / a_{i}^{p} a_{j}^{q}\right)\right)^{1 /(p+q)}} \\
=\operatorname{BHM}^{p, q}\left(a_{1}, a_{2}, \ldots, a_{n}\right) .
\end{aligned}
$$

Example 10. Let $\widehat{a}_{1}=[3,4,6], \widehat{a}_{2}=[1,2,4], \widehat{a}_{3}=[2,4,5]$, $\widehat{a}_{4}=[3,5,6]$, and $\widehat{a}_{5}=[2,5,7]$ be a collection of triangular fuzzy numbers. To rank these triangular fuzzy numbers, we first compare each triangular fuzzy number $\widehat{a}_{i}$ with all triangular fuzzy numbers $\widehat{a}_{j}(j=1,2,3,4,5)$ by using $(7)$ (without loss of generality, set $\delta=0.5)$; let $p_{i j}=p\left(\widehat{a}_{i} \geq\right.$ $\left.\widehat{a}_{j}\right)(i, j=1,2,3,4,5)$, then we utilize these possibility degrees to construct the following matrix $P=\left(p_{i j}\right)_{5 \times 5}$ :

$$
P=\left(\begin{array}{ccccc}
0.500 & 1 & 0.667 & 0.333 & 0.375 \\
0 & 0.500 & 0 & 0 & 0 \\
0.333 & 1 & 0.500 & 0.125 . & 0.200 \\
0.667 & 1 & 0.875 & 0.500 & 0.467 \\
0.625 & 1 & 0.800 & 0.533 & 0.500
\end{array}\right)
$$

Summing all elements in each line of matrix $P$, we have

$$
\begin{gathered}
p_{1}=2.875, \quad p_{2}=0.500, \quad p_{3}=2.158, \\
p_{4}=3.509, \quad p_{5}=3.458 .
\end{gathered}
$$

and then we rank the triangular fuzzy numbers $\widehat{a}_{i}(i=$ $1,2,3,4,5)$ in descending order in accordance with the values of $p_{i}(i=1,2,3,4,5)$ as follows:

$$
\begin{gathered}
\widehat{a}_{\sigma(1)}=\widehat{a}_{4}, \quad \widehat{a}_{\sigma(2)}=\widehat{a}_{5}, \quad \widehat{a}_{\sigma(3)}=\widehat{a}_{1}, \\
\widehat{a}_{\sigma(4)}=\widehat{a}_{3}, \quad \widehat{a}_{\sigma(5)}=\widehat{a}_{2} .
\end{gathered}
$$


Suppose that the weighting vector of the FOWBHM operator is $\omega=(0.1117,0.2365,0.3036,0.2365,0.1117)^{T}$ (derived by the normal distribution based method [27]), and then by (22) (let $p=q=2$ ), we get

$$
\begin{aligned}
& \operatorname{FOWBHM}^{2,2}\left(\widehat{a}_{1}, \hat{a}_{2}, \hat{a}_{3}, \hat{a}_{4}, \hat{a}_{5}\right) \\
& =\left[\frac{1}{\left(\sum_{i, j=1}^{5}\left(\omega_{i} \omega_{j} /\left(a_{\sigma(i)}^{L}\right)^{2}\left(a_{\sigma(j)}^{L}\right)^{2}\right)\right)^{1 / 4}},\right. \\
& \\
& \frac{\left(\sum_{i, j=1}^{5}\left(\omega_{i} \omega_{j} /\left(a_{\sigma(i)}^{M}\right)^{2}\left(a_{\sigma(j)}^{M}\right)^{2}\right)\right)^{1 / 4}}{} \\
& \left.\frac{\left(\sum_{i, j=1}^{5}\left(\omega_{i} \omega_{j} /\left(a_{\sigma(i)}^{U}\right)^{2}\left(a_{\sigma(j)}^{U}\right)^{2}\right)\right)^{1 / 4}}{=}\right] . \\
& =[1.901,3.632,5.509] .
\end{aligned}
$$

Both FWBHM and FOWBHM operators, however, can only deal with the situation in which there are correlations between any two aggregated arguments, but not the situation in which there exist connections among any three aggregated arguments. To solve this issue, motivated by Definition 3, we define the following.

Definition 11. Let $\widehat{a}_{i}=\left[a_{i}^{L}, a_{i}^{M}, a_{i}^{U}\right](i=1,2, \ldots, n)$ be a collection of triangular fuzzy numbers and let $w=$ $\left(w_{1}, w_{2}, \ldots, w_{n}\right)^{T}$ be the weight vector of $\widehat{a}_{i}(i=1,2, \ldots, n)$, where $w_{i}$ indicates the importance degree of $\hat{a}_{i}$, satisfying $w_{i}>0, i=1,2, \ldots, n$ and $\sum_{i=1}^{n} w_{i}=1$. For $p, q, r \geq 0$, if

$$
\begin{aligned}
\operatorname{GFWBHM}^{p, q, r}\left(\widehat{a}_{1}, \widehat{a}_{2}, \ldots, \widehat{a}_{n}\right) \\
=\frac{1}{\left(\sum_{i, j, k=1}^{n}\left(w_{i} w_{j} w_{k} / \widehat{a}_{i}^{p} \widehat{a}_{j}^{q} \widehat{a}_{k}^{r}\right)\right)^{1 /(p+q+r)}} \\
=\left[\frac{1}{\left(\sum_{i, j, k=1}^{n}\left(w_{i} w_{j} w_{k} /\left(a_{i}^{L}\right)^{p}\left(a_{j}^{L}\right)^{q}\left(a_{k}^{L}\right)^{r}\right)\right)^{1 /(p+q+r)},}\right. \\
\\
\quad \frac{1}{\left(\sum_{i, j, k=1}^{n}\left(w_{i} w_{j} w_{k} /\left(a_{i}^{M}\right)^{p}\left(a_{j}^{M}\right)^{q}\left(a_{k}^{M}\right)^{r}\right)\right)^{1 /(p+q+r)}}, \\
\\
\left.\quad \frac{1}{\left(\sum_{i, j, k=1}^{n}\left(w_{i} w_{j} w_{k} /\left(a_{i}^{U}\right)^{p}\left(a_{j}^{U}\right)^{q}\left(a_{k}^{U}\right)^{r}\right)\right)^{1 /(p+q+r)}}\right],
\end{aligned}
$$

then GFWBHM ${ }^{p, q, r}$ is called generalized fuzzy weighted Bonferroni harmonic mean (GFWBHM) operator.
In particular, if $w=(1 / n, 1 / n, \ldots, 1 / n)^{T}$, then the GFWBHM operator reduces to the following:

$$
\begin{aligned}
\operatorname{GFBHM}^{p, q, r}\left(\widehat{a}_{1}, \widehat{a}_{2}, \ldots, \widehat{a}_{n}\right) \\
=\frac{1}{\left(\left(1 / n^{3}\right) \sum_{i, j, k=1}^{n}\left(1 / \widehat{a}_{i}^{p} \widehat{a}_{j}^{q} \widehat{a}_{k}^{r}\right)\right)^{1 /(p+q+r)}},
\end{aligned}
$$

which we call the generalized fuzzy Bonferroni harmonic mean (GFBHM) operator.

In addition, some special cases can be obtained as the change of parameters.

(1) If $r=0$, then the GFWBHM operator reduces to

$$
\begin{aligned}
\operatorname{GFWBHM}^{p, q, 0}\left(\widehat{a}_{1}, \widehat{a}_{2}, \ldots, \widehat{a}_{n}\right) \\
=\frac{1}{\left(\sum_{i, j, k=1}^{n}\left(w_{i} w_{j} w_{k} / \widehat{a}_{i}^{p} \widehat{a}_{j}^{q}\right)\right)^{1 /(p+q)}} \\
=\frac{1}{\left(\left(\sum_{k=1}^{n} w_{k}\right) \sum_{i, j=1}^{n}\left(w_{i} w_{j} / \widehat{a}_{i}^{p} \widehat{a}_{j}^{q}\right)\right)^{1 /(p+q)}} \\
=\frac{1}{\left(\sum_{i, j=1}^{n}\left(w_{i} w_{j} / \widehat{a}_{i}^{p} \widehat{a}_{j}^{q}\right)\right)^{1 /(p+q)}},
\end{aligned}
$$

which is the FWBHM operator.

(2) If $q=0$ and $r=0$, then the GFWBHM operator reduces to

$$
\begin{aligned}
\operatorname{GFWBHM}^{p, 0,0}\left(\widehat{a}_{1}, \widehat{a}_{2}, \ldots, \widehat{a}_{n}\right) \\
=\frac{1}{\left(\sum_{i, j, k=1}^{n}\left(w_{i} w_{j} w_{k} / \widehat{a}_{i}^{p}\right)\right)^{1 / p}} \\
=\frac{1}{\left(\left(\sum_{j=1}^{n} w_{j}\right)\left(\sum_{k=1}^{n} w_{k}\right) \sum_{i=1}^{n}\left(w_{i} / \widehat{a}_{i}^{p}\right)\right)^{1 / p}} \\
=\frac{1}{\left(\sum_{i=1}^{n}\left(w_{i} / \hat{a}_{i}^{p}\right)\right)^{1 / p}},
\end{aligned}
$$

which is FWGHM operator. In this case, if $p=1$, then FWGHM operator reduces to FWHM operator.

Similar to the FWBHM operator, the GFWBHM operator has the following properties.

Theorem 12. Let $p, q, r \geq 0$, and let $\widehat{a}_{i}=\left[a_{i}^{L}, a_{i}^{M}, a_{i}^{U}\right](i=$ $1,2, \ldots, n)$ be a collection of triangular fuzzy numbers, and the following are valid.

(1) Idempotency. If all $\widehat{a}_{i}(i=1,2, \ldots, n)$ are equal, that is, $\widehat{a}_{i}=$ $\hat{a}$, for all $i$, then

$$
\operatorname{GFWBHM}^{p, q, r}\left(\widehat{a}_{1}, \widehat{a}_{2}, \ldots, \widehat{a}_{n}\right)=\widehat{a} .
$$

(2) Boundedness. $\widehat{a}^{-} \leq$GFWBHM $^{p, q, r}\left(\widehat{a}_{1}, \widehat{a}_{2}, \ldots, \widehat{a}_{n}\right) \leq$ $\widehat{a}^{+}$, where $\widehat{a}^{-}=\left[\min _{i}\left\{a_{i}^{L}\right\}, \min _{i}\left\{a_{i}^{M}\right\}, \min _{i}\left\{a_{i}^{U}\right\}\right]$ and $\widehat{a}^{+}=$ $\left[\max _{i}\left\{a_{i}^{L}\right\}, \max _{i}\left\{a_{i}^{M}\right\}, \max _{i}\left\{a_{i}^{U}\right\}\right]$.

$$
\widehat{a}^{+}=\left[\max _{i}\left\{a_{i}^{L}\right\}, \max _{i}\left\{a_{i}^{M}\right\}, \max _{i}\left\{a_{i}^{U}\right\}\right] .
$$


(3) Commutativity. Let $\hat{a}_{i}^{\prime}=\left[a_{i}^{\prime L}, a_{i}^{\prime M}, a_{i}^{\prime U}\right](i=1,2, \ldots, n)$ be a collection of triangular fuzzy numbers, and then

$$
\begin{aligned}
& \operatorname{GFWBHM}^{p, q, r}\left(\widehat{a}_{1}, \widehat{a}_{2}, \ldots, \widehat{a}_{n}\right) \\
& =\operatorname{GFWBHM}^{p, q, r}\left(\hat{a}_{1}^{\prime}, \hat{a}_{2}^{\prime}, \ldots, \hat{a}_{n}^{\prime}\right),
\end{aligned}
$$

where $\left(\hat{a}_{1}^{\prime}, \hat{a}_{2}^{\prime}, \ldots, \hat{a}_{n}^{\prime}\right)$ is any permutation of $\left(\widehat{a}_{1}, \widehat{a}_{2}, \ldots, \widehat{a}_{n}\right)$.

In particular, if the triangular fuzzy numbers $\widehat{a}_{i}=$ $\left[a_{i}^{L}, a_{i}^{M}, a_{i}^{U}\right](i=1,2, \ldots, n)$ reduce to the interval numbers $\tilde{a}_{i}=\left[a_{i}^{L}, a_{i}^{U}\right](i=1,2, \ldots, n)$, then the GFWBHM operator (24) reduces to the generalized uncertain weighted Bonferroni harmonic mean (GUWBHM) operator as follows:

$$
\begin{aligned}
\operatorname{GUWBHM}^{p, q, r}\left(\widetilde{a}_{1}, \tilde{a}_{2}, \ldots, \widetilde{a}_{n}\right) \\
=\frac{1}{\left(\sum_{i, j, k=1}^{n}\left(w_{i} w_{j} w_{k} / \widetilde{a}_{i}^{p} \widetilde{a}_{j}^{q} \widetilde{a}_{k}^{r}\right)\right)^{1 /(p+q+r)}} \\
=\left[\frac{1}{\left(\sum_{i, j, k=1}^{n}\left(w_{i} w_{j} w_{k} /\left(a_{i}^{L}\right)^{p}\left(a_{j}^{L}\right)^{q}\left(a_{k}^{L}\right)^{r}\right)\right)^{1 /(p+q+r)}},\right. \\
\left.\frac{1}{\left(\sum_{i, j, k=1}^{n}\left(w_{i} w_{j} w_{k} /\left(a_{i}^{U}\right)^{p}\left(a_{j}^{U}\right)^{q}\left(a_{k}^{U}\right)^{r}\right)\right)^{1 /(p+q+r)}}\right] .
\end{aligned}
$$

If $w=(1 / n, 1 / n, \ldots, 1 / n)^{T}$, then the GUWBHM operator reduces to the generalized uncertain Bonferroni harmonic mean (GUBHM):

$$
\begin{aligned}
\operatorname{GUBHM}^{p, q, r}\left(\widetilde{a}_{1}, \tilde{a}_{2}, \ldots, \tilde{a}_{n}\right) \\
=\frac{1}{\left(\left(1 / n^{3}\right) \sum_{i, j, k=1}^{n}\left(1 / \tilde{a}_{i}^{p} \widetilde{a}_{j}^{q} \widetilde{a}_{k}^{r}\right)\right)^{1 /(p+q+r)}} \\
=\left[\frac{1}{\left(\left(1 / n^{3}\right) \sum_{i, j, k=1}^{n}\left(1 /\left(a_{i}^{L}\right)^{p}\left(a_{j}^{L}\right)^{q}\left(a_{k}^{L}\right)^{r}\right)\right)^{1 /(p+q+r)}},\right. \\
\left.\frac{1}{\left(\left(1 / n^{3}\right) \sum_{i, j, k=1}^{n}\left(1 /\left(a_{i}^{U}\right)^{p}\left(a_{j}^{U}\right)^{q}\left(a_{k}^{U}\right)^{r}\right)\right)^{1 /(p+q+r)}}\right] .
\end{aligned}
$$

Furthermore, if $a_{i}^{L}=a_{i}^{U}=a_{i}$, for all $i$, then the GUWBHM operator reduces to the generalized weighted Bonferroni harmonic mean (GWBHM) operator:

$$
\begin{aligned}
\operatorname{GWBHM}^{p, q, r}\left(a_{1}, a_{2}, \ldots, a_{n}\right) \\
=\frac{1}{\left(\sum_{i, j, k=1}^{n}\left(w_{i} w_{j} w_{k} / a_{i}^{p} a_{j}^{q} a_{k}^{r}\right)\right)^{1 /(p+q+r)}} .
\end{aligned}
$$

In this case, if $p=1$ and $q=r=0$, the GWBHM operator reduces to the weighted harmonic mean (WHM) operator.
Example 13. Consider the four triangular fuzzy numbers $\widehat{a}_{i}$ and their weight vector $w$ given in Example 8. Then by the GFWBHM operator (33) (without of generalization, let $p=$ $q=r=3$ ), we have

$$
\begin{aligned}
& \operatorname{GFWBHM}^{3,3,3}\left(\widehat{a}_{1}, \widehat{a}_{2}, \widehat{a}_{3}, \widehat{a}_{4}\right) \\
& =\left[\frac{1}{\left(\sum_{i, j, k=1}^{4}\left(w_{i} w_{j} w_{k} /\left(a_{i}^{L}\right)^{3}\left(a_{j}^{L}\right)^{3}\left(a_{k}^{L}\right)^{3}\right)\right)^{1 / 9}},\right. \\
& \\
& \qquad \frac{1}{\left(\sum_{i, j, k=1}^{4}\left(w_{i} w_{j} w_{k} /\left(a_{i}^{M}\right)^{3}\left(a_{j}^{M}\right)^{3}\left(a_{k}^{M}\right)^{3}\right)\right)^{1 / 9}}, \\
& =[1.21,2.89,4.59] .
\end{aligned}
$$

Definition 14. Let $\widehat{a}_{i}=\left[a_{i}^{L}, a_{i}^{M}, a_{i}^{U}\right](i=1,2, \ldots, n)$ be a collection of triangular fuzzy numbers. For $p, q, r \geq 0$, a generalized fuzzy ordered weighted Bonferroni harmonic mean (GFOWBHM) operator of dimension $n$ is a mapping GFOWBHM $^{p, q, r}: \Omega^{n} \rightarrow \Omega$, that has an associated vector $\omega=\left(\omega_{1}, \omega_{2}, \ldots, \omega_{n}\right)^{T}$ such that $\omega_{i} \geq 0$ and $\sum_{i=1}^{n} \omega_{i}=1$. Furthermore,

$$
\begin{aligned}
& \operatorname{GFOWBHM}^{p, q, r}\left(\widehat{a}_{1}, \widehat{a}_{2}, \ldots, \widehat{a}_{n}\right) \\
& =\frac{1}{\left(\sum_{i, j, k=1}^{n}\left(\omega_{i} \omega_{j} \omega_{k} / \hat{a}_{\sigma(i)}^{p} \widehat{a}_{\sigma(j)}^{q} \widehat{a}_{\sigma(k)}^{r}\right)\right)^{1 /(p+q+r)}} \\
& =\left[\frac{1}{\left(\sum_{i, j, k=1}^{n}\left(\omega_{i} \omega_{j} \omega_{k} /\left(a_{\sigma(i)}^{L}\right)^{p}\left(a_{\sigma(j)}^{L}\right)^{q}\left(a_{\sigma(k)}^{L}\right)^{r}\right)\right)^{1 /(p+q+r)}},\right. \\
& \frac{1}{\left(\sum_{i, j, k=1}^{n}\left(\omega_{i} \omega_{j} \omega_{k} /\left(a_{\sigma(i)}^{M}\right)^{p}\left(a_{\sigma(j)}^{M}\right)^{q}\left(a_{\sigma(k)}^{M}\right)^{r}\right)\right)^{1 /(p+q+r)}}, \\
& \left.\frac{1}{\left(\sum_{i, j, k=1}^{n}\left(\omega_{i} \omega_{j} \omega_{k} /\left(a_{\sigma(i)}^{U}\right)^{p}\left(a_{\sigma(j)}^{U}\right)^{q}\left(a_{\sigma(k)}^{U}\right)^{r}\right)\right)^{1 /(p+q+r)}}\right],
\end{aligned}
$$

where $\widehat{a}_{\sigma(i)}=\left[a_{\sigma(i)}^{L}, a_{\sigma(i)}^{M}, a_{\sigma(i)}^{U}\right](i=1,2, \ldots, n)$, and $(\sigma(1)$, $\sigma(2), \ldots, \sigma(n))$ is a permutation of $(1,2, \ldots, n)$ such that $\widehat{a}_{\sigma(i-1)} \geq \widehat{a}_{\sigma(i)}$ for all $i$.

However, if there is a tie between $\widehat{a}_{i}$ and $\widehat{a}_{j}$, then we replace each of $\widehat{a}_{i}$ and $\widehat{a}_{j}$ by their average $\left(\widehat{a}_{i}+\widehat{a}_{j}\right) / 2$ in process of aggregation. If $k$ items are tied, then we replace these by $k$ replicas of their average. 
If $\omega=(1 / n, 1 / n, \ldots, 1 / n)^{T}$, then the GFOWBHM operator reduces to the GFBHM operator. Moreover, some special cases can be obtained as the change of parameters. If $r=0$, then the GFOWBHM operator reduces to FOWBHM operator; if $r=0$ and $q=0$, then GFOWBHM operator reduces to FOWGHM operator. In particular, if the triangular fuzzy numbers $\widehat{a}_{i}=\left[a_{i}^{L}, a_{i}^{M}, a_{i}^{U}\right](i=1,2, \ldots, n)$ reduce to the interval numbers $\widetilde{a}_{i}=\left[a_{i}^{L}, a_{i}^{U}\right](i=1,2, \ldots, n)$, then the GFOWBHM operator reduces to the generalized uncertain ordered weighted Bonferroni harmonic mean (GUOWBHM) operator:

$$
\begin{aligned}
& \operatorname{GUOWBHM}^{p, q, r}\left(\widetilde{a}_{i}, \widetilde{a}_{2}, \ldots, \widetilde{a}_{n}\right) \\
& =\frac{1}{\left(\sum_{i, j, k=1}^{n}\left(\omega_{i} \omega_{j} \omega_{k} / \tilde{a}_{\sigma(i)}^{p} \widetilde{a}_{\sigma(j)}^{q} \widetilde{a}_{\sigma(k)}^{r}\right)\right)^{1 /(p+q+r)}} \\
& =\left[\frac{1}{\left(\sum_{i, j, k=1}^{n}\left(\omega_{i} \omega_{j} \omega_{k} /\left(a_{\sigma(i)}^{L}\right)^{p}\left(a_{\sigma(j)}^{L}\right)^{q}\left(a_{\sigma(k)}^{L}\right)^{r}\right)\right)^{1 /(p+q+r)}},\right. \\
& \left.\frac{1}{\left(\sum_{i, j, k=1}^{n}\left(\omega_{i} \omega_{j} \omega_{k} /\left(a_{\sigma(i)}^{U}\right)^{p}\left(a_{\sigma(j)}^{U}\right)^{q}\left(a_{\sigma(k)}^{U}\right)^{r}\right)\right)^{1 /(p+q+r)}}\right],
\end{aligned}
$$

where $\tilde{a}_{\sigma(i)}=\left[a_{\sigma(i)}^{L}, a_{\sigma(i)}^{U}\right]$, and $(\sigma(1), \sigma(2), \ldots, \sigma(n))$ is a permutation of $(1,2, \ldots, n)$ such that $\widetilde{a}_{\sigma(i-1)} \geq \widetilde{a}_{\sigma(i)}$ for all $i$.

If $a_{i}^{L}=a_{i}^{U}=a_{i}$, for all $i=1,2, \ldots, n$, then the GUOWBHM operator reduces to the generalized ordered weighted Bonferroni harmonic mean (GOWBHM) operator:

$$
\begin{aligned}
\operatorname{GOWBHM}^{p, q, r}\left(a_{1}, a_{2}, \ldots, a_{n}\right) \\
=\frac{1}{\left(\sum_{i, j, k=1}^{n}\left(\omega_{i} \omega_{j} \omega_{k} / b_{i}^{p} b_{j}^{q} b_{k}^{r}\right)\right)^{1 /(p+q+r)}},
\end{aligned}
$$

where $b_{i}$ is the $i$ th largest of $a_{i}(i=1,2, \ldots, n)$. In this case, if $p=1$ and $q=r=0$, then the GOWBHM operator reduces to the ordered weighted harmonic mean (OWHM) operator.

The GOWBHM operator (46) has some special cases.

(1) If $\omega=(1,0, \ldots, 0)^{T}$, then

$$
\operatorname{GOWBHM}^{p, q, r}\left(a_{1}, a_{2}, \ldots, a_{n}\right)=\max \left\{a_{i}\right\}=b_{1} \text {. }
$$

(2) If $\omega=(0,0, \ldots, 1)^{T}$, then

$$
\operatorname{GOWBHM}^{p, q, r}\left(a_{1}, a_{2}, \ldots, a_{n}\right)=\min \left\{a_{i}\right\}=b_{n} \text {. }
$$

(3) If $\omega=(1 / n, 1 / n, \ldots, 1 / n)^{T}$, then

$$
\begin{aligned}
\operatorname{GOWBHM}^{p, q, r}\left(a_{1}, a_{2}, \ldots, a_{n}\right) \\
=\frac{1}{\left(\left(1 / n^{3}\right) \sum_{i, j, k=1}^{n}\left(1 / b_{i}^{p} b_{j}^{q} b_{k}^{r}\right)\right)^{1 /(p+q+r)}} \\
=\frac{1}{\left(\left(1 / n^{3}\right) \sum_{i, j, k=1}^{n}\left(1 / a_{i}^{p} a_{j}^{q} a_{k}^{r}\right)\right)^{1 /(p+q+r)}},
\end{aligned}
$$

which we call the generalized Bonferroni harmonic mean (GBHM) operator.

Example 15. Consider the four triangular fuzzy numbers $\widehat{a}_{i}$ and their weight vector $w$ given in Example 10. Then by the GFOWBHM operator (44) (let $p=q=r=3$ ), we have

$$
\begin{aligned}
& \operatorname{GFOWBHM}^{3,3,3}\left(\widehat{a}_{1}, \widehat{a}_{2}, \widehat{a}_{3}, \widehat{a}_{4}, \widehat{a}_{5}\right) \\
& =\frac{1}{\left(\sum_{i, j, k=1}^{5}\left(w_{i} w_{j} w_{k} /\left(a_{\sigma(i)}^{L}\right)^{3}\left(a_{\sigma(j)}^{L}\right)^{3}\left(a_{\sigma(k)}^{L}\right)^{3}\right)\right)^{1 / 9}}, \\
& \frac{1}{\left(\sum_{i, j, k=1}^{5}\left(w_{i} w_{j} w_{k} /\left(a_{\sigma(i)}^{M}\right)^{3}\left(a_{\sigma(j)}^{M}\right)^{3}\left(a_{\sigma(k)}^{M}\right)^{3}\right)\right)^{1 / 9}}, \\
& =[1.751,3.410,5.422] .
\end{aligned}
$$

In the following section, we will apply the developed operators to multiple attribute group decision making.

\section{An Approach to Multiple Attribute Group Decision Making with Triangular Fuzzy Information}

For a group decision making with triangular fuzzy information, let $X=\left\{x_{1}, x_{2}, \ldots, x_{n}\right\}$ be a discrete set of $n$ alternatives, let $G=\left\{G_{1}, G_{2}, \ldots, G_{m}\right\}$ be the set of $m$ attributes, whose weight vector is $w=\left(w_{1}, w_{2}, \ldots, w_{m}\right)^{T}$ with $w_{i} \geq 0$ and $\sum_{i=1}^{m} w_{i}=1$, and let $D=\left\{d_{1}, d_{2}, \ldots, d_{s}\right\}$ be the set of decision makers, whose weight vector is $v=\left(v_{1}, v_{2}, \ldots, v_{s}\right)^{T}$, where $v_{k} \geq 0$ and $\sum_{k=1}^{s} v_{k}=1$. Suppose that $A^{(k)}=\left(\hat{a}_{i j}^{(k)}\right)_{m \times n}$ is the decision matrix, where $\widehat{a}_{i j}^{(k)}=\left[a_{i j}^{L(k)}, a_{i j}^{M(k)}, a_{i j}^{U(k)}\right]$ is an attribute value, which takes the form of triangular fuzzy number, of the alternative $x_{j} \in X$ with respect to the attribute $G_{i} \in G$.

In the following, we apply the GFWBHM and GFOWBHM operators to group decision making with triangular fuzzy information.

Step 1. Normalize each attribute value $\widehat{a}_{i j}^{(k)}$ in the matrix $A^{(k)}$ into a corresponding element in the matrix $R^{(k)}=\left(\widehat{r}_{i j}^{(k)}\right)_{m \times n}$ $\left(\widehat{r}_{i j}^{(k)}=\left[r_{i j}^{L(k)}, r_{i j}^{M(k)}, r_{i j}^{U(k)}\right]\right)$ using the following formulas:

$\widehat{r}_{i j}^{(k)}=\frac{\widehat{a}_{i j}^{(k)}}{\sum_{j=1}^{n} \hat{a}_{i j}^{(k)}}$ 


$$
=\left[\frac{a_{i j}^{L(k)}}{\sum_{j=1}^{n} a_{i j}^{U(k)}}, \frac{a_{i j}^{M(k)}}{\sum_{j=1}^{n} a_{i j}^{M(k)}}, \frac{a_{i j}^{U(k)}}{\sum_{j=1}^{n} a_{i j}^{L(k)}}\right],
$$

for benefit attribute $G_{i}, i=1,2, \ldots, m$,

$$
\begin{gathered}
j=1,2, \ldots, n, k=1,2, \ldots, s, \\
\widehat{r}_{i j}^{(k)}=\frac{1 / \widehat{a}_{i j}^{(k)}}{\sum_{j=1}^{n}\left(1 / \widehat{a}_{i j}^{(k)}\right)} \\
=\left[\frac{1 / a_{i j}^{U(k)}}{\sum_{j=1}^{n}\left(1 / a_{i j}^{L(k)}\right)}, \frac{1 / a_{i j}^{M(k)}}{\sum_{j=1}^{n}\left(1 / a_{i j}^{M(k)}\right)}, \frac{1 / a_{i j}^{L(k)}}{\sum_{j=1}^{n}\left(1 / a_{i j}^{U(k)}\right)}\right],
\end{gathered}
$$

for cost attribute $G_{i}, i=1,2, \ldots, m$,

$$
j=1,2, \ldots, n, k=1,2, \ldots, s .
$$

Step 2. Utilize the GFWBHM operator (33) as follows:

$$
\begin{aligned}
\widehat{r}_{j}^{(k)}=\operatorname{GFWBHM}^{p, q, r}\left(\widehat{r}_{1 j}^{(k)}, \widehat{r}_{2 j}^{(k)}, \ldots, \widehat{r}_{m j}^{(k)}\right) & \frac{1}{\left(\sum_{i, h, l=1}^{m}\left(w_{i} w_{h} w_{l} /\left(\widehat{r}_{i j}^{(k)}\right)^{p}\left(\widehat{r}_{h j}^{(k)}\right)^{q}\left(\widehat{r}_{l j}^{(k)}\right)^{r}\right)\right)^{1 /(p+q+r)}} \\
= & {\left[\sum_{i, h, l=1}^{m}\left(\frac{w_{i} w_{h} w_{l}}{\left(r_{i j}^{L(k)}\right)^{p}\left(r_{h j}^{L(k)}\right)^{q}\left(r_{l j}^{L(k)}\right)^{r}}\right)\right)^{-1 /(p+q+r)} } \\
& \left(\sum_{i, h, l=1}^{m}\left(\frac{w_{i} w_{h} w_{l}}{\left(r_{i j}^{M(k)}\right)^{p}\left(r_{h j}^{M(k)}\right)^{q}\left(r_{l j}^{M(k)}\right)^{r}}\right)\right)^{-1 /(p+q+r)}, \\
& \left.\left(\sum_{i, h, l=1}^{m}\left(\frac{w_{i} w_{h} w_{l}}{\left(r_{i j}^{U(k)}\right)^{p}\left(r_{h j}^{U(k)}\right)^{q}\left(r_{l j}^{U(k)}\right)^{r}}\right)\right)^{-1 /(p+q+r)}\right],
\end{aligned}
$$

to aggregate all the elements in the $j$ th column of $R^{(k)}$ and get the overall attribute value $\hat{r}_{j}^{(k)}$ of the alternative $x_{j}$ corresponding to the decision maker $d_{k}$.

Step 3. Utilize the GFOWBHM operator (44):

$$
\begin{aligned}
\widehat{r}_{j} & =\operatorname{GFOWBHM}^{p, q, r}\left(\widehat{r}_{j}^{(1)}, \widehat{r}_{j}^{(2)}, \ldots, \widehat{r}_{j}^{(s)}\right) \\
& =\frac{1}{\left(\sum_{k, h, l=1}^{s}\left(\omega_{k} \omega_{h} \omega_{l} /\left(\dot{\hat{r}}_{j}^{\sigma(k)}\right)^{p}\left(\dot{\hat{r}}_{j}^{\sigma(h)}\right)^{q}\left(\dot{\hat{r}}_{j}^{\sigma(l)}\right)^{r}\right)\right)^{1 /(p+q+r)}} \\
& =\left[\left(\sum_{k, h, l=1}^{s}\left(\frac{\omega_{k} \omega_{h} \omega_{l}}{\left(\dot{r}_{j}^{L(\sigma(k))}\right)^{p}\left(\dot{r}_{j}^{L(\sigma(h))}\right)^{q}\left(\dot{r}_{j}^{L(\sigma(l))}\right)^{r}}\right)\right)^{-1 /(p+q+r)}\right.
\end{aligned}
$$

$$
\begin{gathered}
\left(\sum_{k, h, l=1}^{s}\left(\frac{\omega_{k} \omega_{h} \omega_{l}}{\left(\dot{r}_{j}^{M(\sigma(k))}\right)^{p}\left(\dot{r}_{j}^{M(\sigma(h))}\right)^{q}\left(\dot{r}_{j}^{M(\sigma(l))}\right)^{r}}\right)\right)^{-1 /(p+q+r)}, \\
\left.\left(\sum_{k, h, l=1}^{s}\left(\frac{\omega_{k} \omega_{h} \omega_{l}}{\left(\dot{r}_{j}^{U(\sigma(k))}\right)^{p}\left(\dot{r}_{j}^{U(\sigma(h))}\right)^{q}\left(\dot{r}_{j}^{U(\sigma(l))}\right)^{r}}\right)\right)^{-1 /(p+q+r)}\right],
\end{gathered}
$$

to aggregate the overall attribute values $\widehat{r}_{j}^{(k)}(k=1,2, \ldots, s)$ corresponding to the decision maker $d_{k}(k=1,2, \ldots, s)$ and get the collective overall attribute value $\widehat{r}_{j}$, where $\dot{\hat{r}}_{j}^{(\sigma(k))}=$ $\left[\dot{r}_{j}^{L(\sigma(k))}, \dot{r}_{j}^{M(\sigma(k))}, \dot{r}_{j}^{U(\sigma(k))}\right]$ is the $k$ th largest of the weighted data and $\dot{\hat{r}}_{j}^{(k)}\left(\dot{\hat{r}}_{j}^{(k)}=s v_{k} \widehat{r}_{j}^{(k)}, k=1,2, \ldots, s\right), \omega=\left(\omega_{1}, \omega_{2}\right.$, $\left.\ldots, \omega_{s}\right)^{T}$ is the weighting vector of the GFOWBHM operator, with $\omega_{k} \geq 0$ and $\sum_{k=1}^{s} \omega_{k}=1$.

Step 4. Compare each $\widehat{r}_{j}$ with all $\widehat{r}_{i}(i=1,2, \ldots, n)$ by using (7), and let $p_{i j}=p\left(\widehat{r}_{i} \geq \widehat{r}_{j}\right)$, and then construct the possibility matrix $P=\left(p_{i j}\right)_{n \times n}$, where $p_{i j} \geq 0, p_{i j}+p_{j i}=1, p_{i i}=0.5$, $i, j=1,2, \ldots, n$. Summing all elements in each line of matrix $P$, we have $p_{i}=\sum_{j=1}^{n} p_{i j}, i=1,2, \ldots, n$, and then reorder $\widehat{r}_{j}(j=1,2, \ldots, n)$ in descending order in accordance with the values of $p_{j}(j=1,2, \ldots, n)$.

Step 5. Rank all alternatives $x_{j}(j=1,2, \ldots, n)$ by the ranking of $\widehat{r}_{j}(j=1,2, \ldots, n)$, and then select the most desirable one.

Step 6. End.

\section{Example Illustrations}

In this section, we use a multiple attribute group decision making problem of determining what kind of airconditioning systems should be installed in a library (adopted from $[21,30])$ to illustrate the proposed approach.

A city is planning to build a municipal library. One of the problems facing the city development commissioner is to determine what kind of air-conditioning systems should be installed in the library. The contractor offers five feasible alternatives, which might be adapted to the physical structure of the library. The alternatives $x_{j}(j=1,2,3,4,5)$ are to be evaluated using triangular fuzzy numbers by the three decision makers $d_{k}(k=1,2,3)$ (whose weight vector is $\left.v=(0.4,0.3,0.3)^{T}\right)$ under three major impacts: economic, functional, and operational. Two monetary attributes and six nonmonetary attributes (i.e., $G_{1}$ : owning cost $\left(\$ / \mathrm{ft}^{2}\right)$, $G_{2}$ : operating cost $\left(\$ / \mathrm{ft}^{2}\right), G_{3}$ : performance $\left({ }^{*}\right), G_{4}$ : noise level (Db), $G_{5}$ : maintainability $\left({ }^{*}\right), G_{6}$ : reliability (\%), $G_{7}$ : flexibility $\left({ }^{*}\right), G_{8}$ : safety $\left({ }^{*}\right)$, where ${ }^{*}$ unit is from 0 to 1 scale, three attributes $G_{1}, G_{2}$, and $G_{4}$ are cost attributes, and the other five attributes are benefit attributes, and suppose that the weight vector of the attributes $G_{i}(i=1,2, \ldots, 8)$ is $\left.w=(0.05,0.08,0.14,0.12,0.18,0.21,0.05,0.17)^{T}\right)$ emerged from three impacts is Tables 1,2 , and 3 .

In the following, we utilize the decision procedure to select the best air-conditioning system. 
TABLE 1: Triangular fuzzy number decision matrix $A^{(1)}$.

\begin{tabular}{cccccc}
\hline & $x_{1}$ & $x_{2}$ & $x_{3}$ & $x_{4}$ & $x_{5}$ \\
\hline$G_{1}$ & {$[3.5,4.0,4.7]$} & {$[1.7,2.0,2.3]$} & {$[3.5,3.8,4.2]$} & {$[3.5,3.8,4.5]$} & {$[3.3,3.8,4.0]$} \\
$G_{2}$ & {$[5.5,6.0,6.5]$} & {$[4.8,5.1,5.5]$} & {$[4.5,5.2,5.5]$} & {$[4.5,4.7,5.0]$} & {$[5.5,5.7,6.0]$} \\
$G_{3}$ & {$[0.7,0.8,0.9]$} & {$[0.5,0.56,0.6]$} & {$[0.5,0.6,0.7]$} & {$[0.7,0.85,0.9]$} & {$[0.6,0.7,0.8]$} \\
$G_{4}$ & {$[35,40,45]$} & {$[70,73,75]$} & {$[65,68,70]$} & {$[40,42,45]$} & {$[50,55,60]$} \\
$G_{5}$ & {$[0.4,0.45,0.5]$} & {$[0.4,0.44,0.6]$} & {$[0.7,0.76,0.8]$} & {$[0.9,0.97,1.0]$} & {$[0.5,0.54,0.6]$} \\
$G_{6}$ & {$[95,98,100]$} & {$[70,73,75]$} & {$[80,83,90]$} & {$[90,93,95]$} & {$[85,90,95]$} \\
$G_{7}$ & {$[0.3,0.35,0.5]$} & {$[0.7,0.75,0.8]$} & {$[0.8,0.9,1.0]$} & {$[0.6,0.75,0.8]$} & {$[0.4,0.5,0.6]$} \\
$G_{8}$ & {$[0.7,0.74,0.8]$} & {$[0.5,0.53,0.6]$} & {$[0.6,0.68,0.7]$} & {$[0.7,0.8,0.9]$} & {$[0.8,0.85,0.9]$} \\
\hline
\end{tabular}

TABLE 2: Triangular fuzzy number decision matrix $A^{(2)}$.

\begin{tabular}{lccccc}
\hline & $x_{1}$ & $x_{2}$ & $x_{3}$ & $x_{4}$ & $x_{5}$ \\
\hline$G_{1}$ & {$[4.0,4.3,4.5]$} & {$[2.1,2.2,2.4]$} & {$[5.0,5.1,5.2]$} & {$[4.3,4.4,4.5]$} & {$[3.0,3.3,3.5]$} \\
$G_{2}$ & {$[6.0,6.3,6.5]$} & {$[5.0,5.1,5.2]$} & {$[4.5,4.7,5.0]$} & {$[5.0,5.1,5.3]$} & {$[7.0,7.5,8.0]$} \\
$G_{3}$ & {$[0.7,0.8,0.9]$} & {$[0.4,0.5,0.6]$} & {$[0.5,0.55,0.6]$} & {$[0.7,0.75,0.8]$} & {$[0.7,0.8,0.9]$} \\
$G_{4}$ & {$[37,38,39]$} & {$[70,73,75]$} & {$[65,66,67]$} & {$[40,42,45]$} & {$[50,52,55]$} \\
$G_{5}$ & {$[0.4,0.5,0.6]$} & {$[0.5,0.55,0.6]$} & {$[0.8,0.85,0.9]$} & {$[0.8,0.95,1.0]$} & {$[0.4,0.44,0.5]$} \\
$G_{6}$ & {$[92,93,95]$} & {$[70,75,80]$} & {$[83,84,85]$} & {$[90,91,92]$} & {$[90,93,95]$} \\
$G_{7}$ & {$[0.4,0.45,0.5]$} & {$[0.8,0.85,0.9]$} & {$[0.7,0.73,0.8]$} & {$[0.7,0.85,0.9]$} & {$[0.4,0.45,0.5]$} \\
$G_{8}$ & {$[0.6,0.7,0.8]$} & {$[0.6,0.65,0.7]$} & {$[0.5,0.6,0.7]$} & {$[0.7,0.76,0.8]$} & {$[0.7,0.8,0.9]$} \\
\hline
\end{tabular}

TABLE 3: Triangular fuzzy number decision matrix $A^{(3)}$.

\begin{tabular}{cccccc}
\hline & $x_{1}$ & $x_{2}$ & $x_{3}$ & $x_{4}$ & $x_{5}$ \\
\hline$G_{1}$ & {$[4.3,4.4,4.6]$} & {$[2.2,2.4,2.5]$} & {$[4.5,4.8,5.0]$} & {$[4.7,4.9,5.0]$} & {$[3.1,3.2,3.4]$} \\
$G_{2}$ & {$[6.4,6.7,7.0]$} & {$[5.0,5.2,5.5]$} & {$[4.7,4.8,4.9]$} & {$[5.5,5.7,6.0]$} & {$[6.0,6.5,7.0]$} \\
$G_{3}$ & {$[0.8,0.85,0.9]$} & {$[0.5,0.6,0.7]$} & {$[0.6,0.7,0.8]$} & {$[0.7,0.8,0.9]$} & {$[0.7,0.75,0.8]$} \\
$G_{4}$ & {$[36,38,40]$} & {$[72,73,75]$} & {$[67,68,70]$} & {$[45,48,50]$} & {$[55,57,60]$} \\
$G_{5}$ & {$[0.4,0.46,0.5]$} & {$[0.4,0.45,0.6]$} & {$[0.8,0.95,1.0]$} & {$[0.8,0.85,0.9]$} & {$[0.5,0.55,0.6]$} \\
$G_{6}$ & {$[93,94,95]$} & {$[77,78,80]$} & {$[85,87,90]$} & {$[90,94,95]$} & {$[90,96,100]$} \\
$G_{7}$ & {$[0.4,0.5,0.6]$} & {$[0.8,0.9,1.0]$} & {$[0.8,0.86,0.9]$} & {$[0.6,0.7,0.8]$} & {$[0.5,0.57,0.6]$} \\
$G_{8}$ & {$[0.7,0.78,0.8]$} & {$[0.5,0.55,0.6]$} & {$[0.6,0.68,0.7]$} & {$[0.8,0.85,0.9]$} & {$[0.8,0.85,0.9]$} \\
\hline
\end{tabular}

Step 1. By using (51), we normalize each attribute value $\widehat{a}_{i j}^{(k)}$ in the matrices $A^{(k)}(k=1,2,3)$ into the corresponding element in the matrices $R^{(k)}=\left(\widehat{r}_{i j}\right)_{8 \times 5}(k=1,2,3)$ (Tables 4,5 , and 6).

Step 2. Utilize the GFWBHM operator (52) (let $p=q=r=3$ ) to aggregate all elements in the $j$ th column $R^{(k)}$ and get the overall attribute value $\widehat{r}_{j}^{(k)}$ :

$$
\begin{aligned}
& \widehat{r}_{1}^{(1)}=[0.1390,0.1753,0.2187], \\
& \widehat{r}_{2}^{(1)}=[0.1347,0.1586,0.1927], \\
& \widehat{r}_{3}^{(1)}=[0.1581,0.1852,0.2178], \\
& \widehat{r}_{4}^{(1)}=[0.1900,0.2289,0.2651], \\
& \widehat{r}_{5}^{(1)}=[0.1565,0.1911,0.2311], \\
& \widehat{r}_{1}^{(2)}=[0.1480,0.1851,0.2248],
\end{aligned}
$$

$\widehat{r}_{2}^{(2)}=[0.1434,0.1706,0.1992]$,

$\widehat{r}_{3}^{(2)}=[0.1561,0.1792,0.2057]$,

$\widehat{r}_{4}^{(2)}=[0.1927,0.2228,0.2477]$,

$\widehat{r}_{5}^{(2)}=[0.1499,0.1761,0.2098]$,

$\widehat{r}_{1}^{(3)}=[0.1459,0.1811,0.2104]$,

$\widehat{r}_{2}^{(3)}=[0.1370,0.1607,0.1938]$,

$\widehat{r}_{3}^{(3)}=[0.1679,0.1921,0.2173]$,

$\widehat{r}_{4}^{(3)}=[0.1883,0.2138,0.2395]$,

$\widehat{r}_{5}^{(3)}=[0.1678,0.1922,0.2215]$. 
TABLE 4: Normalized triangular fuzzy number decision matrix $R^{(1)}$.

\begin{tabular}{cccccc}
\hline & $x_{1}$ & $x_{2}$ & $x_{3}$ & $x_{4}$ & $x_{5}$ \\
\hline$G_{1}$ & {$[0.12,0.16,0.21]$} & {$[0.25,0.32,0.43]$} & {$[0.14,0.17,0.21]$} & {$[0.13,0.17,0.21]$} & {$[0.14,0.17,0.22]$} \\
$G_{2}$ & {$[0.15,0.18,0.21]$} & {$[0.18,0.21,0.24]$} & {$[0.18,0.20,0.25]$} & {$[0.20,0.23,0.25]$} & {$[0.16,0.19,0.21]$} \\
$G_{3}$ & {$[0.18,0.23,0.30]$} & {$[0.13,0.16,0.20]$} & {$[0.13,0.17,0.23]$} & {$[0.18,0.24,0.30]$} & {$[0.15,0.20,0.27]$} \\
$G_{4}$ & {$[0.22,0.26,0.32]$} & {$[0.13,0.14,0.16]$} & {$[0.14,0.15,0.17]$} & {$[0.22,0.25,0.28]$} & {$[0.16,0.19,0.23]$} \\
$G_{5}$ & {$[0.11,0.14,0.17]$} & {$[0.11,0.14,0.21]$} & {$[0.20,0.24,0.28]$} & {$[0.26,0.31,0.34]$} & {$[0.14,0.17,0.21]$} \\
$G_{6}$ & {$[0.21,0.22,0.24]$} & {$[0.15,0.17,0.18]$} & {$[0.18,0.19,0.21]$} & {$[0.20,0.21,0.23]$} & {$[0.19,0.21,0.23]$} \\
$G_{7}$ & {$[0.08,0.11,0.18]$} & {$[0.19,0.23,0.29]$} & {$[0.22,0.28,0.36]$} & {$[0.16,0.23,0.29]$} & {$[0.11,0.15,0.21]$} \\
$G_{8}$ & {$[0.18,0.21,0.24]$} & {$[0.13,0.15,0.18]$} & {$[0.15,0.19,0.21]$} & {$[0.18,0.22,0.27]$} & {$[0.21,0.24,0.27]$} \\
\hline
\end{tabular}

TABLE 5: Normalized triangular fuzzy number decision matrix $R^{(2)}$.

\begin{tabular}{cccccc}
\hline & $x_{1}$ & $x_{2}$ & $x_{3}$ & $x_{4}$ & $x_{5}$ \\
\hline$G_{1}$ & {$[0.15,0.16,0.19]$} & {$[0.28,0.32,0.36]$} & {$[0.13,0.14,0.15]$} & {$[0.15,0.16,0.17]$} & {$[0.19,0.21,0.25]$} \\
$G_{2}$ & {$[0.17,0.18,0.19]$} & {$[0.21,0.22,0.23]$} & {$[0.21,0.24,0.26]$} & {$[0.20,0.22,0.23]$} & {$[0.13,0.15,0.17]$} \\
$G_{3}$ & {$[0.18,0.24,0.30]$} & {$[0.11,0.15,0.20]$} & {$[0.13,0.16,0.20]$} & {$[0.18,0.22,0.27]$} & {$[0.18,0.24,0.30]$} \\
$G_{4}$ & {$[0.25,0.27,0.29]$} & {$[0.13,0.14,0.15]$} & {$[0.15,0.15,0.16]$} & {$[0.22,0.24,0.27]$} & {$[0.18,0.20,0.21]$} \\
$G_{5}$ & {$[0.11,0.15,0.21]$} & {$[0.14,0.17,0.21]$} & {$[0.22,0.26,0.31]$} & {$[0.22,0.29,0.34]$} & {$[0.11,0.13,0.17]$} \\
$G_{6}$ & {$[0.21,0.21,0.22]$} & {$[0.16,0.17,0.19]$} & {$[0.19,0.19,0.20]$} & {$[0.20,0.21,0.22]$} & {$[0.20,0.21,0.22]$} \\
$G_{7}$ & {$[0.11,0.14,0.17]$} & {$[0.22,0.26,0.30]$} & {$[0.19,0.22,0.27]$} & {$[0.19,0.26,0.30]$} & {$[0.19,0.14,0.17]$} \\
$G_{8}$ & {$[0.15,0.20,0.26]$} & {$[0.15,0.19,0.23]$} & {$[0.13,0.17,0.23]$} & {$[0.18,0.22,0.26]$} & {$[0.18,0.23,0.29]$} \\
\hline
\end{tabular}

TABLE 6: Normalized triangular fuzzy number decision matrix $R^{(3)}$.

\begin{tabular}{lccccc}
\hline & $x_{1}$ & $x_{2}$ & $x_{3}$ & $x_{4}$ & $x_{5}$ \\
\hline$G_{1}$ & {$[0.15,0.17,0.18]$} & {$[0.28,0.30,0.35]$} & {$[0.14,0.15,0.17]$} & {$[0.14,0.15,0.16]$} & {$[0.20,0.23,0.25]$} \\
$G_{2}$ & {$[0.16,0.17,0.19]$} & {$[0.20,0.22,0.24]$} & {$[0.22,0.24,0.25]$} & {$[0.18,0.20,0.22]$} & {$[0.16,0.17,0.20]$} \\
$G_{3}$ & {$[0.20,0.23,0.27]$} & {$[0.12,0.16,0.21]$} & {$[0.15,0.19,0.24]$} & {$[0.17,0.22,0.27]$} & {$[0.17,0.20,0.24]$} \\
$G_{4}$ & {$[0.26,0.28,0.31]$} & {$[0.14,0.15,0.16]$} & {$[0.15,0.16,0.17]$} & {$[0.21,0.22,0.25]$} & {$[0.17,0.19,0.20]$} \\
$G_{5}$ & {$[0.11,0.14,0.17]$} & {$[0.11,0.14,0.21]$} & {$[0.22,0.29,0.34]$} & {$[0.22,0.26,0.31]$} & {$[0.14,0.17,0.21]$} \\
$G_{6}$ & {$[0.20,0.21,0.22]$} & {$[0.17,0.17,0.18]$} & {$[0.18,0.19,0.21]$} & {$[0.20,0.21,0.22]$} & {$[0.20,0.21,0.23]$} \\
$G_{7}$ & {$[0.10,0.14,0.19]$} & {$[0.21,0.25,0.32]$} & {$[0.21,0.24,0.29]$} & {$[0.15,0.20,0.26]$} & {$[0.13,0.16,0.19]$} \\
$G_{8}$ & {$[0.18,0.21,0.24]$} & {$[0.13,0.15,0.18]$} & {$[0.15,0.18,0.21]$} & {$[0.21,0.23,0.26]$} & {$[0.21,0.23,0.26]$} \\
\hline
\end{tabular}

Step 3. Utilize the GFOWBHM operator (53) (suppose that its weight vector is $\omega=(0.243,0.514,0.243)^{T}$ determined by using the normal distribution based method [27] (let $\delta=0.5$ and $p=q=r=3$ ) to aggregate the overall attribute value $\widehat{r}_{j}^{(k)}(k=1,2,3)$, corresponding to the decision maker $d_{k}(k=$ $1,2,3)$, and get the collective overall attribute value $\widehat{r}_{j}$ :

$$
\begin{aligned}
& \widehat{r}_{1}=[0.1459,0.1816,0.2195], \\
& \widehat{r}_{2}=[0.1395,0.1649,0.1962], \\
& \widehat{r}_{3}=[0.1592,0.1837,0.2111], \\
& \widehat{r}_{4}=[0.1909,0.2218,0.2493], \\
& \widehat{r}_{5}=[0.1552,0.1830,0.2171] .
\end{aligned}
$$

Step 4. Compare each $\widehat{r}_{j}$ with all $\widehat{r}_{i}(i=1,2,3,4,5)$ by using (7) (without loss of generality, set $\delta=0.5)$, and let $p_{i j}=p\left(\widehat{r}_{i} \geq\right.$ $\widehat{r}_{j}$ ), and then construct a possibility matrix:

$$
P=\left(\begin{array}{ccccc}
0.5 & 0.7387 & 0.4598 & 0 & 0.4610 \\
0.2613 & 0.5 & 0.1638 & 0 & 0.1921 \\
0.5402 & 0.8362 & 0.5 & 0 & 0.5010 \\
1 & 1 & 1 & 0.5 & 1 \\
0.5390 & 0.8079 & 0.4990 & 0 & 0.5
\end{array}\right)
$$

Summing all elements in each line of matrix $P$, we have

$$
\begin{gathered}
p_{1}=2.1595, \quad p_{2}=1.1171, \quad p_{3}=2.3774, \\
p_{4}=4.5, \quad p_{5}=2.3459,
\end{gathered}
$$

and then reorder $\widehat{r}_{j}(j=1,2,3,4,5)$ in descending order in accordance with the values of $p_{j}(j=1,2,3,4,5)$ :

$$
\widehat{r}_{4}>\widehat{r}_{3}>\widehat{r}_{5}>\widehat{r}_{1}>\widehat{r}_{2}
$$

Step 5. Rank all the alternatives $x_{j}(j=1,2,3,4,5)$ by the ranking of $\widehat{r}_{j}(j=1,2,3,4,5)$ :

$$
x_{4}>x_{3}>x_{5}>x_{1}>x_{2},
$$

and thus the most desirable alternative is $x_{4}$.

From the previous analysis, the results obtained by the proposed approach are very similar to the ones obtained Xu's approach [21], but our approach is more flexible than that of $\mathrm{Xu}$ [21] because it can provide the decision makers more choices as parameters are assigned different values. 
TABLE 7: Comparison of the proposed approach with other approaches.

\begin{tabular}{|c|c|c|c|c|}
\hline & Xu’s approach [21] & Wei's approach [22] & $\begin{array}{l}\text { Sun and Sun's approach } \\
{[23]}\end{array}$ & Proposed approach \\
\hline Problem type & MAGDM & MAGDM & MADM & MAGDM \\
\hline Application area & $\begin{array}{l}\text { Air-conditioning system } \\
\text { selection }\end{array}$ & Investment of money & EPR system selection & $\begin{array}{l}\text { Air-conditioning system } \\
\text { selection }\end{array}$ \\
\hline Decision information & $\begin{array}{l}\text { Triangular fuzzy decision } \\
\text { matrix }\end{array}$ & $\begin{array}{l}\text { Triangular fuzzy } \\
\text { decision matrix }\end{array}$ & $\begin{array}{l}\text { Triangular fuzzy decision } \\
\text { matrix }\end{array}$ & $\begin{array}{c}\text { Triangular fuzzy decision } \\
\text { matrix }\end{array}$ \\
\hline \multicolumn{5}{|l|}{ Solution method } \\
\hline Aggregation stage & FWHM operator & FIOWHM operator & & GFWBHM operator \\
\hline Exploitation stage & FHHM operator & FWHM operator & FOBHM operator & GFOWBHM operator \\
\hline Ranking stage & Complementary matrix & Complementary matrix & Possibility matrix & Possibility matrix \\
\hline Final decision & Ranking of alternatives & Ranking of alternatives & Ranking of alternatives & Ranking of alternatives \\
\hline
\end{tabular}

\section{Comparison of the Proposed Approach with Other Approaches}

In this section, we compare the proposed approach with other approaches. The approaches to be compared here are the approaches proposed by $\mathrm{Xu}$ [21], Wei [22], and H. Sun and M. Sun [23], respectively.

Each of methods has its advantages and disadvantages and none of them can always perform better than the others in any situations. It perfectly depends on how we look at things and not on how they are themselves. The differences in four approaches are the following.

(1) The H. Sun and M. Sun's approach is only suitable for solving multiple attribute decision making (MADM), while the proposed approach and Xu's and Wei's approaches are suitable for solving MAGDM because the approaches provide the aggregation stage in aggregation process.

(2) The Xu's and Wei's approaches have simple computation process than the proposed approach and H. Sun and M. Sun's approach, while the proposed approach and H. Sun and M. Sun's approach are more flexible than Xu's and Wei's approaches because these can provide the decision makers more choices as parameters are assigned different values.

(3) The Wei's approach uses the weights of decision makers as the order inducing variables in aggregation stage, while other approaches use the weights of decision makers to determine the order positions of the overall attribute values in exploitation stage.

Others of relative comparison with Xu's, Wei's, and H. Sun and M. Sun's approaches are shown in Table 7.

\section{Conclusions}

In this paper, we have extended the GWBM operator to the triangular fuzzy environment and developed the fuzzy harmonic aggregation operators including the FWBHM and GFWBHM operators. Based on the these operators and Yager's OWA operator, we have developed the FOWBHM operator and the GFOWBHM operator, respectively, and discussed their properties and special cases. It has been pointed out that if all the input fuzzy data reduce to the interval or numerical data, then the GFWBHM operator reduces to the GUWBHM operator and GWBHM operator, respectively; the GFOWBHM operator reduces to the GUOWBHM operator and GOWBHM operator, respectively. In these situations, the WHM (resp., OWHM) operator is the special case of the GWBHM (resp. GOWBHM) operator. Based on the GFWBHM and GFOWBHM operators, we have developed an approach to multiple attribute group decision making with triangular fuzzy information and have also applied the proposed approach to the problem of determining what kind of air-conditioning systems should be installed in the library. Furthermore, the comparison of the proposed approach with other existing approaches is presented. The merit of the proposed approach is that it is more flexible than the classical ones because it can provide the decision makers more choices as parameters are assigned different values. Apparently, the proposed aggregation techniques and decision making method can also extended to the intervalvalued triangular fuzzy environment.

\section{Acknowledgment}

This work was supported by a Research Grant of Pukyong National University (2013).

\section{References}

[1] R. R. Yager, "On ordered weighted averaging aggregation operators in multicriteria decisionmaking," IEEE Transactions on Systems, Man, and Cybernetics, vol. 18, no. 1, pp. 183-190, 1988.

[2] R. R. Yager, "Families of OWA operators," Fuzzy Sets and Systems, vol. 59, no. 2, pp. 125-148, 1993.

[3] F. Chiclana, F. Herrera, and E. Herrera-Viedma, "The ordered weighted geometric operator: properties and application," in Proceedings of the 8th International Conference on Information Processing and Management of Uncertainty in Knowledge-Based Systems, pp. 985-991, Madrid, Spain, 2000. 
[4] Z. S. Xu and Q. L. Da, "The ordered weighted geometric averaging operators," International Journal of Intelligent Systems, vol. 17, no. 7, pp. 709-716, 2002.

[5] Z. S. Xu and Q. L. Da, "An overview of operators for aggregating information," International Journal of Intelligent Systems, vol. 18, no. 9, pp. 953-969, 2003.

[6] H. Y. Chen, C. L. Liu, and Z. H. Shen, "Induced ordered weighted harmonic averaging (IOWHA) operator and its application to combination forecasting method," Chinese Journal of Management Science, vol. 12, pp. 35-40, 2004.

[7] R. R. Yager, "OWA aggregation over a continuous interval argument with applications to decision making," IEEE Transactions on Systems, Man, and Cybernetics B, vol. 34, no. 5, pp. 1952-1963, 2004.

[8] R. R. Yager and Z. Xu, "The continuous ordered weighted geometric operator and its application to decision making," Fuzzy Sets and Systems, vol. 157, no. 10, pp. 1393-1402, 2006.

[9] R. R. Yager, "Choquet aggregation using order inducing variables," International Journal of Uncertainty, Fuzziness and Knowledge-Based Systems, vol. 12, no. 1, pp. 69-88, 2004.

[10] R. R. Yager, “The power average operator," IEEE Transactions on Systems, Man, and Cybernetics A, vol. 31, no. 6, pp. 724-731, 2001.

[11] Z. Xu and R. R. Yager, "Power-geometric operators and their use in group decision making," IEEE Transactions on Fuzzy Systems, vol. 18, no. 1, pp. 94-105, 2010.

[12] C. Bonferroni, "Sulle medie multiple di potenze," Bollettino dell'Unione Matematica Italiana, vol. 5, no. 3-4, pp. 267-270, 1950.

[13] R. R. Yager, "On generalized Bonferroni mean operators for multi-criteria aggregation," International Journal of Approximate Reasoning, vol. 50, no. 8, pp. 1279-1286, 2009.

[14] G. Beliakov, S. James, J. Mordelová, T. Rückschlossová, and R. R. Yager, "Generalized Bonferroni mean operators in multicriteria aggregation," Fuzzy Sets and Systems, vol. 161, no. 17, pp. 2227-2242, 2010.

[15] M. Xia, Z. Xu, and B. Zhu, "Generalized intuitionistic fuzzy Bonferroni means," International Journal of Intelligent Systems, vol. 27, no. 1, pp. 23-47, 2012.

[16] C. Tan and X. Chen, "Intuitionistic fuzzy Choquet integral operator for multi-criteria decision making," Expert Systems with Applications, vol. 37, no. 1, pp. 149-157, 2010.

[17] Z. Xu, "Choquet integrals of weighted intuitionistic fuzzy information," Information Sciences, vol. 180, no. 5, pp. 726-736, 2010.

[18] Z. Xu and R. R. Yager, "Intuitionistic fuzzy bonferroni means," IEEE Transactions on Systems, Man, and Cybernetics B, vol. 41, no. 2, pp. 568-578, 2011.

[19] D. Yu, Y. Wu, and W. Zhou, "Generalized hesitant fuzzy bonferroni mean and its application in multi-criteria group decision making," Journal of Information and Computational Science, vol. 9, no. 2, pp. 267-274, 2012.

[20] Z. Xu, "Approaches to multiple attribute group decision making based on intuitionistic fuzzy power aggregation operators," Knowledge-Based Systems, vol. 24, no. 6, pp. 749-760, 2011.

[21] Z. Xu, "Fuzzy harmonic mean operators," International Journal of Intelligent Systems, vol. 24, no. 2, pp. 152-172, 2009.

[22] G.-W. Wei, "FIOWHM operator and its application to multiple attribute group decision making," Expert Systems with Applications, vol. 38, no. 4, pp. 2984-2989, 2011.
[23] H. Sun and M. Sun, "Generalized Bonferroni harmonic mean operators and their application to multiple attribute decision making," Journal of Computational Information Systems, vol. 8, no. 14, pp. 5717-5724, 2012.

[24] P. J. M. van Laarhoven and W. Pedrycz, "A fuzzy extension of Saaty's priority theory," Fuzzy Sets and Systems, vol. 11, no. 3, pp. 229-241, 1983.

[25] F. Chiclana, F. Herrera, and E. Herrera-Viedma, "Integrating multiplicative preference relations in a multipurpose decisionmaking model based on fuzzy preference relations," Fuzzy Sets and Systems, vol. 122, no. 2, pp. 277-291, 2001.

[26] Z. S. Xu and Q. L. Da, “The uncertain OWA operator," International Journal of Intelligent Systems, vol. 17, no. 6, pp. 569-575, 2002.

[27] Z. Xu, "An overview of methods for determining OWA weights," International Journal of Intelligent Systems, vol. 20, no. 8, pp. 843-865, 2005.

[28] R. R. Yager, “Centered OWA operators," Soft Computing, vol. 11, no. 7, pp. 631-639, 2007.

[29] X. Liu and S. Han, "Orness and parameterized RIM quantifier aggregation with OWA operators: a summary," International Journal of Approximate Reasoning, vol. 48, no. 1, pp. 77-97, 2008.

[30] K. Yoon, "The propagation of errors in multiple-attribute decision analysis: a practical approach," Journal of the Operational Research Society, vol. 40, pp. 681-686, 1989. 


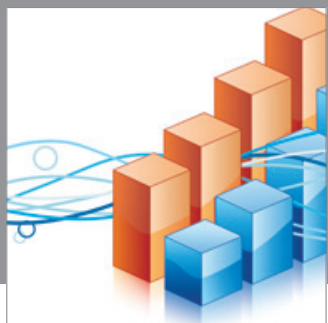

Advances in

Operations Research

mansans

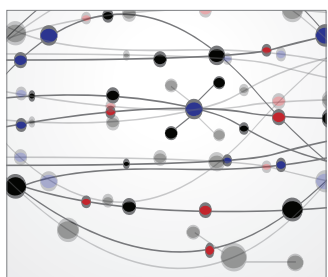

The Scientific World Journal
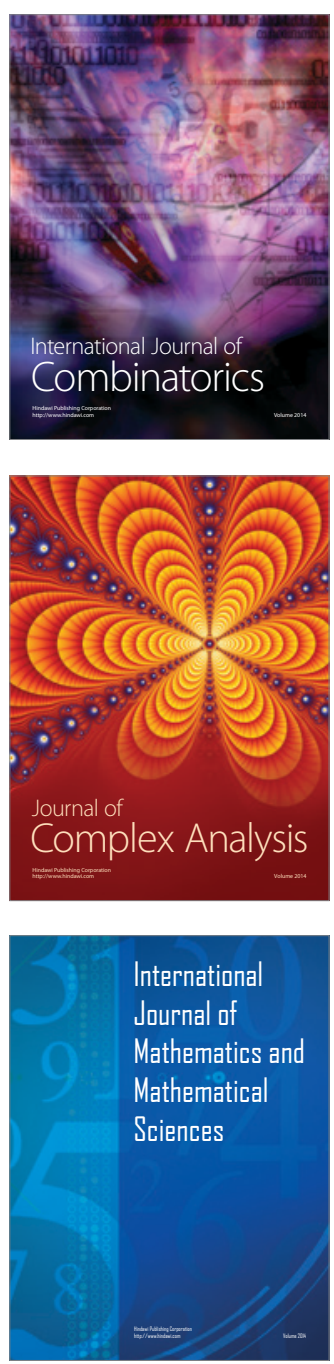
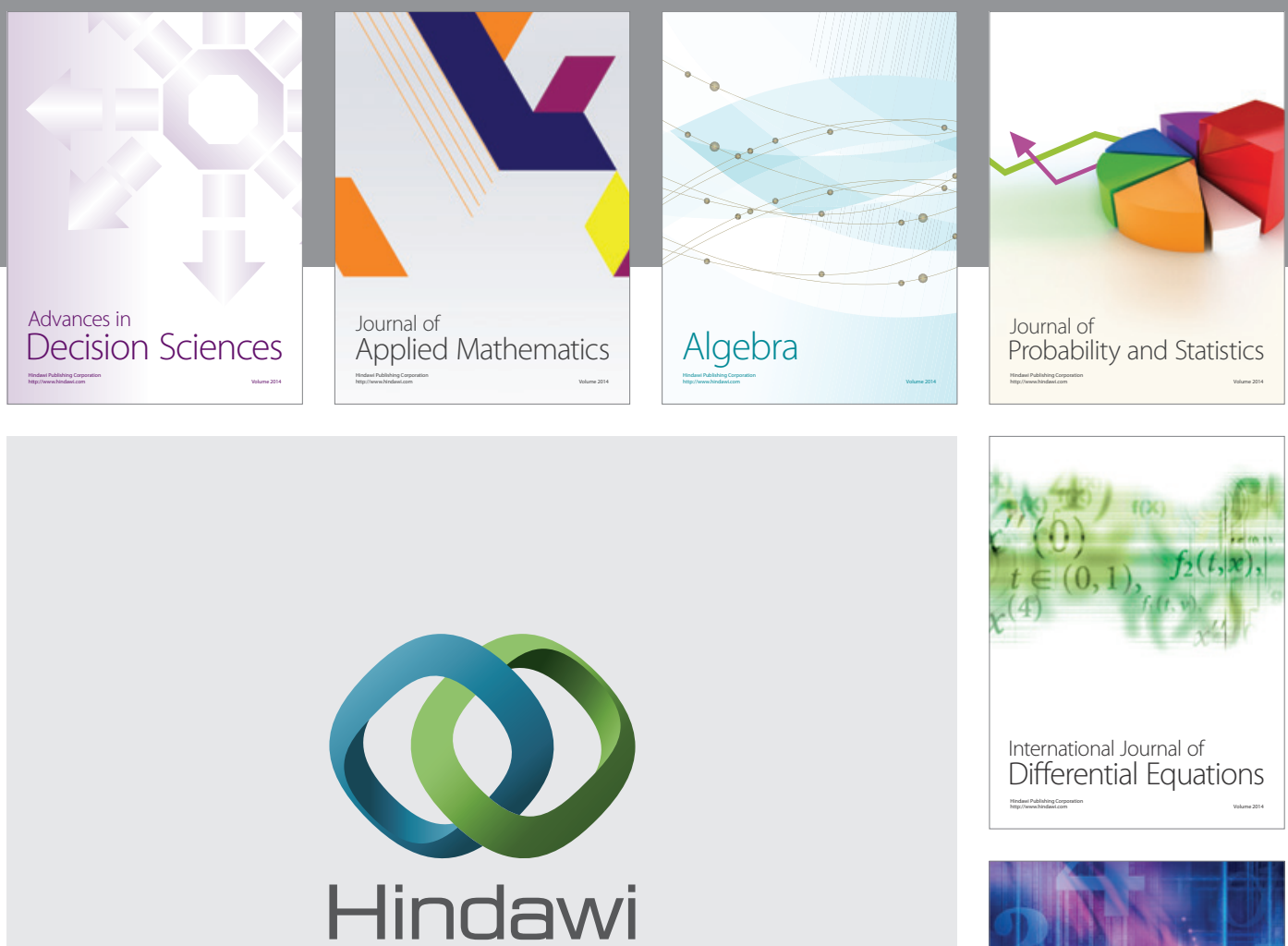

Submit your manuscripts at http://www.hindawi.com
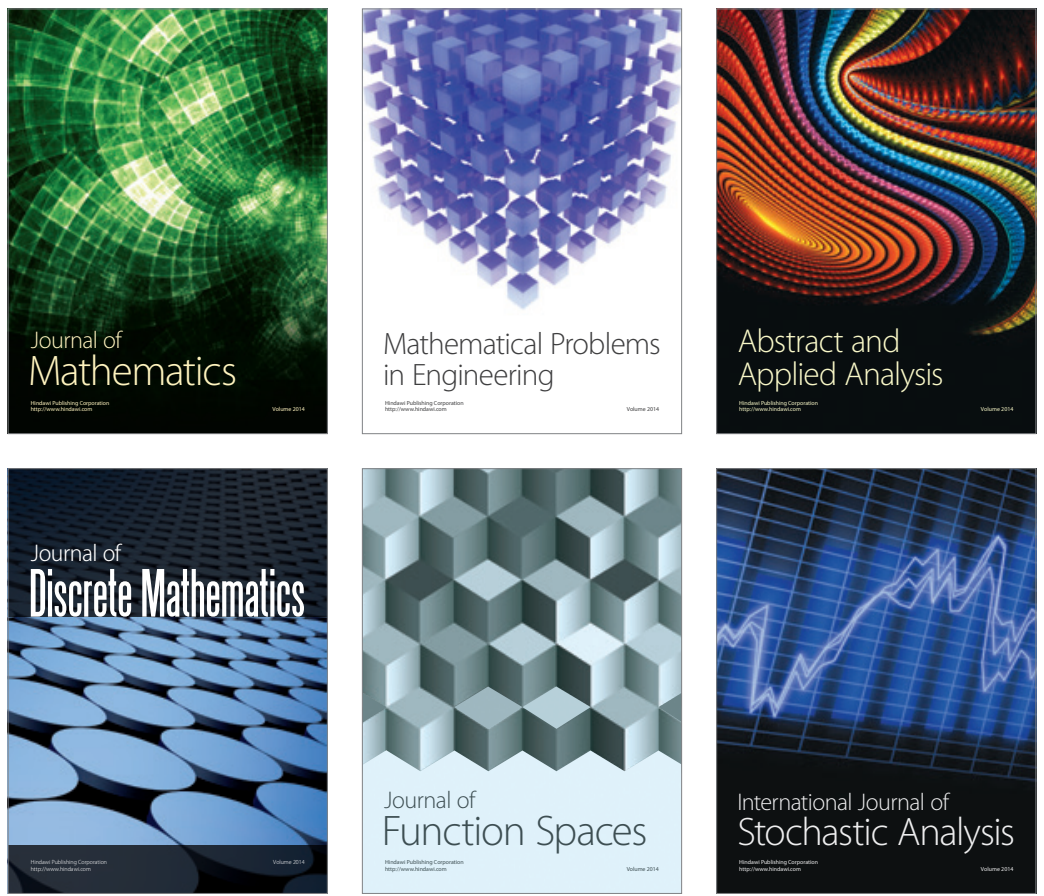

Journal of

Function Spaces

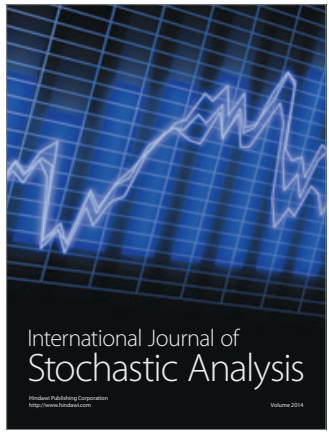

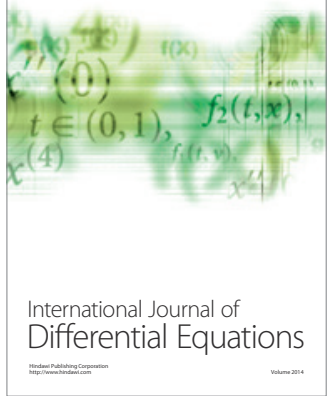
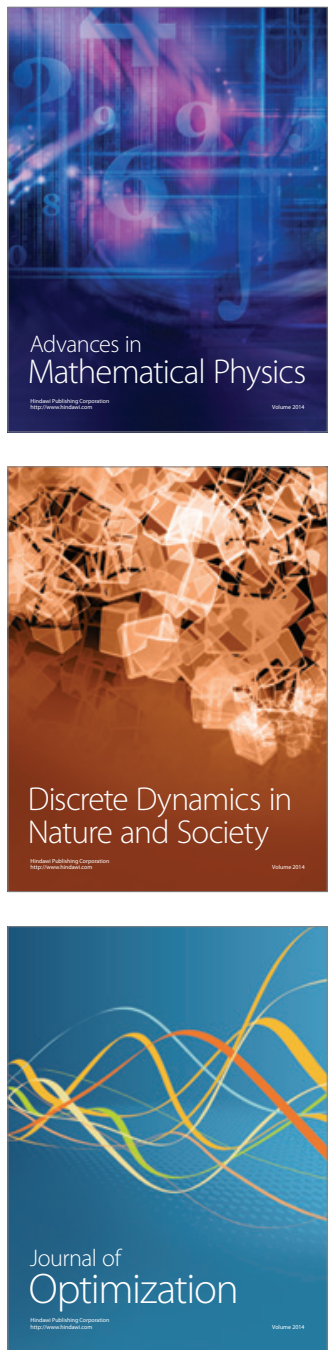